\title{
Article \\ Evaluating the Impact of Different Symmetrical Models of Ambient Assisted Living Systems
}

\author{
Wael Alosaimi ${ }^{1}$, Md Tarique Jamal Ansari ${ }^{2}{ }^{-1}$, Abdullah Alharbi ${ }^{1}$, Hashem Alyami ${ }^{3}$, Adil Hussain Seh ${ }^{4}$, \\ Abhishek Kumar Pandey ${ }^{4}{ }^{\oplus}$, Alka Agrawal ${ }^{4}$ and Raees Ahmad Khan ${ }^{4, *}$ \\ 1 Department of Information Technology, College of Computers and Information Technology, Taif University, \\ P.O. Box 11099, Taif 21944, Saudi Arabia; w.osaimi@tu.edu.sa (W.A.); amharbi@tu.edu.sa (A.A.) \\ 2 Department of Computer Application, Integral University, Lucknow 226026, India; tjtjansari@gmail.com \\ 3 Department of Computer Science, College of Computers and Information Technology, Taif University, \\ P.O. Box 11099, Taif 21944, Saudi Arabia; hyami@tu.edu.sa \\ 4 Department of Information Technology, Babasaheb Bhimrao Ambedkar University, Lucknow 226025, India; \\ ahseh.rs@bbau.ac.in (A.H.S.); abhishekkumarpanday5@gmail.com (A.K.P.); dralka@bbau.ac.in (A.A.) \\ * Correspondence: khanraees@bbau.ac.in or khanraees@yahoo.com
}

Citation: Alosaimi, W.; Ansari, M.T.J.; Alharbi, A.; Alyami, H.; Seh, A.H.; Pandey, A.K.; Agrawal, A.; Khan, R.A. Evaluating the Impact of Different Symmetrical Models of Ambient Assisted Living Systems. Symmetry 2021, 13, 450. https:// doi.org/10.3390/sym13030450

Academic Editor: Jan Awrejcewicz

Received: 10 February 2021

Accepted: 6 March 2021

Published: 10 March 2021

Publisher's Note: MDPI stays neutral with regard to jurisdictional claims in published maps and institutional affiliations.

Copyright: (C) 2021 by the authors Licensee MDPI, Basel, Switzerland. This article is an open access article distributed under the terms and conditions of the Creative Commons Attribution (CC BY) license (https:// creativecommons.org/licenses/by/ $4.0 /)$.

\begin{abstract}
In recent years, numerous attempts have been made to enhance the living standard for old-aged people. Ambient Assisted Living (AAL) is an evolving interdisciplinary field aimed at the exploitation of knowledge and communication technology in health and tele-monitoring systems to combat the impact of the growing aging population. AAL systems are designed for customized, responsive, and predictive requirements, requiring high performance of functionality to ensure interoperability, accessibility, security, and consistency. Standardization, continuity, and assistance of system development have become an urgent necessity to meet the increasing needs for sustainable systems. In this article, we examine and address the methods of the different AAL systems. In addition, we analyzed the acceptance criteria of the AAL framework intending to define and evaluate different AAL-based symmetrical models, leveraging performance characteristics under the integrated fuzzy environment. The main goal is to provide an understanding of the current situation of the AAL-oriented setups. Our vision is to investigate and evaluate the potential symmetrical models of AAL systems and frameworks for the implementation of effective new installations.
\end{abstract}

Keywords: ambient assisted living; AAL; ambient intelligence; assisted living; user-interfaces; fuzzy logic

\section{Introduction}

The current digital environment, comprising smart home products, mobile devices, smart watches, and software applications, has had a significant impact on human lifestyles. These systems have provided a great deal of power to individuals, thereby significantly lowering dependence on others. These advanced devices have not only transformed lifestyles, but have also revolutionized almost every area of human existence. The idea of Ambient Assisted Living (AAL) resulted from these smart technologies, and represents the response to the task of maintaining the standard of living of elderly people. Ambient Assisted Living (AAL) offers a system consisting of smart phones, medical sensors, cellular networks, computers, and health tracking apps [1]. AAL can also be used for different reasons, such as preventing, treating, and enhancing the well-being and health of elderly people.

AAL strives to promote the protection and wellbeing of elderly people and to increase the number of years that elderly people can live comfortably in an area of their own convenience [2-5]. It also reduces the amount of anticipated costs by empowering patients to monitor their serious medical symptoms. AAL is a sub-part of Ambient Intelligence that includes the utilization of ambient intelligent strategies, processes, and technology to allow aged people to survive comfortably for as long as humanly possible despite behavioral problems. 
In addition, modern developments in mobile and portable sensors have contributed to realizing the vision of AAL [6,7]. Recent popular electronic applications are fitted with smart configurations, such as accelerometers, navigation systems, GPS, and many other systems that can be used to monitor user mobility. Furthermore, recent developments in digital and sensor technology are promising a new age in health sensors [8]. Scientists and researchers have created discreet sensors in the form of covers, small holter-type gadgets, mobile systems, and smart clothing for tracking health indicators. For instance, blood sugar, blood pressure and heart performance can be evaluated by means of smart technologies such as infrared or photographic sensors. Many measures, such as electroencephalography (EEG), also involve invasive devices, including electrodes. The following Figure 1 shows the graphical representation of an Ambient Assisted Living (AAL) system.

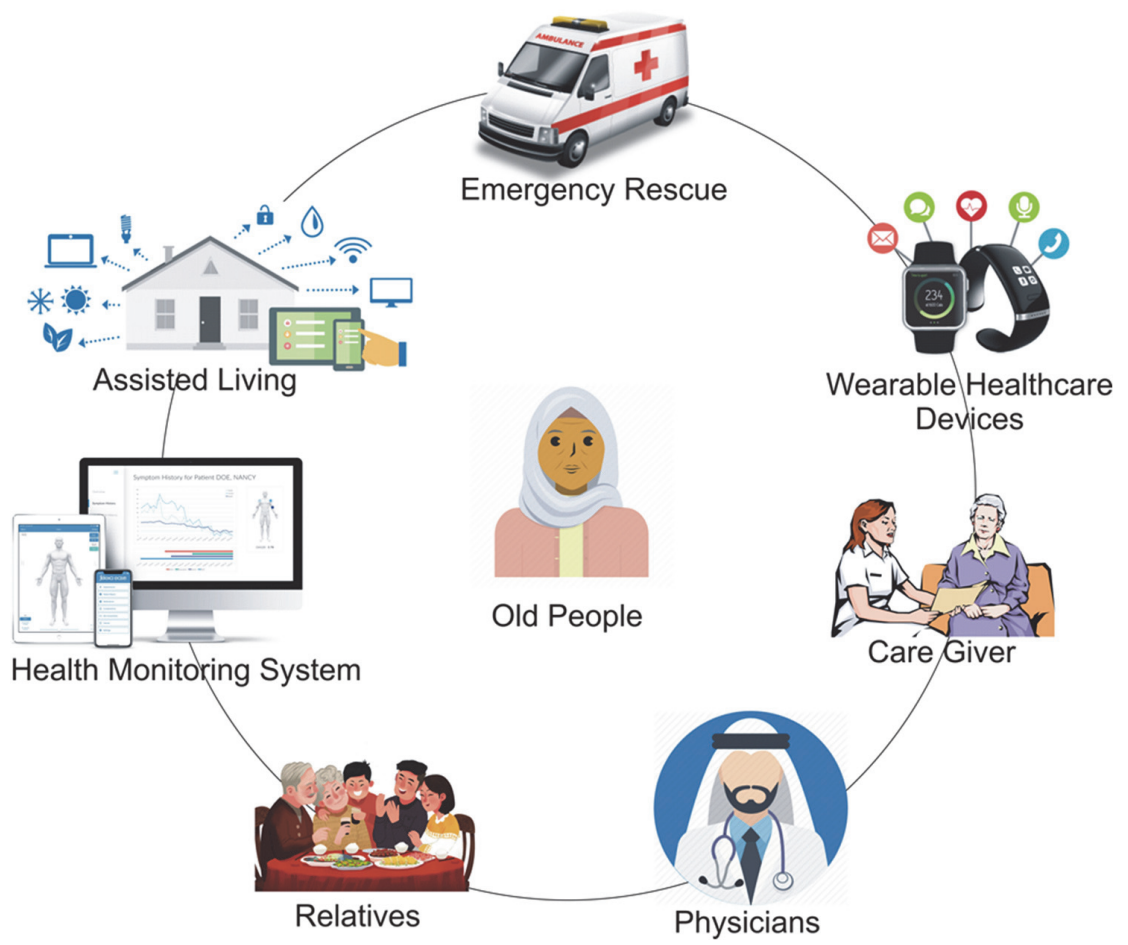

Figure 1. Graphical representation of an Ambient Assisted Living (AAL) system.

This study evaluates different Ambient Assisted Living system's symmetrical models based on the taxonomy adopted from Amina et al. [9]. The study uses integrated fuzzy Analytic Hierarchy Process-Technique for Order Performance by Similarity to Ideal Solution (AHP-TOPSIS), which is a popular multiple-criteria decision-making (MCDM) approach. To handle the complexity of evaluating the efficiency of various Ambient Assisted Living System Symmetrical Models on one parameter, or on the value of another high-precision parameter, the Analytic Hierarchy Process (AHP) method has been extensively used by numerous authors and practitioners. Ghodsypour and O'Brien [10] claim that AHP is much more reliable than other symmetrical models of scoring for analysis procedures. Conceivably, the technique is suitable because the decision-making process has a one-way hierarchical relationship between decision-making stages. Interestingly, Carney and Wallnau [11] noted that the selection parameters for alternatives are not necessarily independent of each other, but rather interconnect. In such a complicated setting, an incorrect result may be obtained. TOPSIS (Technique for Order Performance by Similarity to Idea Solution) [12] is also an appropriate approach for solving MCDM problems. TOPSIS is initiated on the principle that the optimum alternative should also have the smallest distance from the positive idea solution (PIS), and the greatest distance from the negative idea solution. The principle of TOPSIS is logical and comprehensible, and the related calculation is straightfor- 
ward. Consequently, it is important to note the inherent complexity of specifying accurate subjective opinions to the parameters.

In the subsequent sections of this article, we classify the discussion into five sections. Section 2 presents the different related works. Section 3 discusses the materials and methods used in this paper. Section 4 presents the statistical results and evaluates their quality characteristics according to different metrics. Finally, Section 5 concludes the paper.

\section{Related Works}

Fuzzy multiple-criteria decision-making (MCDM) provides successful outcomes in the resolution of selection-based problems [13]. The approach has been chosen by several researchers because it can effectively handle the knowledge that is analyzed using a multi-resourced linguistic and quantitative decision-making challenge, and evidence that is diverse.

Lin et al. [14] suggested a fuzzy-based strategy for the selection of an effective smart technology framework for fall detection. To address this problem, a fuzzy collective intelligence methodology was used. Alpha-cut procedures were used in a fuzzy collective intelligence method to determine the fuzzy weights of the parameters with each decisionmaker. Then, the fuzzy combination was used to sum the fuzzy weights generated by each decision-maker. Consequently, a fuzzy order preference strategy comparable to the ideal solution was used to assess the appropriateness of a smart technology system for fall detection.

Samanlioglu et al. [15] proposed an approach to choosing the best employee applicant for an IT organization by combining the fuzzy analytic hierarchical process method (fuzzy AHP), including Chang's scale analysis, in addition to fuzzy TOPSIS. The decisionmakers' (DMs) verbal assessments were included in the analysis using intuitive fuzzy numbers. They first calculated the value of thirty sub-criteria weights using fuzzy AHP and then, using fuzzy TOPSIS, five IT employees' alternatives were evaluated based on fuzzy AHP weights.

Anand and Vinodh [16] presented research to evaluate Additive Manufacturing (AM) procedures for micro-manufacturing using combined fuzzy AHP-TOPSIS. Parameters weights were derived using fuzzy AHP, whereas rankings were obtained using fuzzy TOPSIS.

Nazam et al. [17] suggested hybrid fuzzy AHP analysis to measure the weight of threat parameters and sub-criteria, in addition to the order efficiency technique using the ideal solution (TOPSIS) procedure to rank and evaluate the risks resulting from the adoption of green supply chain management (GSCM) practices in a fuzzy setting. Their proposed fuzzy risk-oriented assessment theory was implemented for the realistic case of the textile automotive industry. Ultimately, the conceptual model allows academics and professionals to consider the value of performing effective risk assessments while introducing green supply chain interventions.

Ansari et al. [18] addressed the viewpoint of security professionals on the relative value of parameters for the selection of an appropriate Security Requirements Engineering (SRE) system using the multi-criteria decision-making approach. The research was designed and conducted to determine the most suitable SRE approach for quality and stable application development based on the expertise and experience of the security specialist. The hierarchical analysis was performed using a fuzzy TOPSIS method. The efficient SRE selection process was evaluated in pairs.

Kumar et al. [19] used an approach that involves the combination of fuzzy AHP with fuzzy TOPSIS processes to determine the effect of various malware detection techniques in the context of web applications. This research used different variants of a university's software system to test the effect of a variety of current malware detection techniques.

Alenezi et al. [20] used the combined approach of fuzzy AHP-TOPSIS to test security architecture strategies and their attributes. The efficacy of this method was also evaluated on the real-time software system of BBAU, Lucknow, India. In addition, various university web apps were also used to support the findings produced. 
The uncertainty associated with Alzheimer's disease complicates the development of patient monitoring software. To address this problem, Coronato described a systematic approach to modelling and developing intelligent Ambient Assisted Living Systems to monitor the behavior of people with cognitive impairments. The major aspect was an automated method to detect irrational behavior, in addition to a technique for the design of secure AAL systems specifically aimed at individuals with cognitive or mental illnesses. The author also implemented their approach for modelling a monitoring system that can demonstrate feasibility through the identification of abnormal circumstances [21].

Koleva et al. highlighted the main obstacles in formulating and executing an efficient AAL system based on their expertise in the eWALL project. They also provided suggestions for solutions to these obstacles [22].

Many surveys and reviews have been conducted that address the functionality of different models of Ambient Assisted Living systems. However, the fuzzy AHP-TOPSIS approach has not been used to evaluate the impact of different symmetrical models of Ambient Assisted Living systems.

\section{Materials and Methods}

Recent developments in a variety of technical fields have helped to realize the potential of AAL. These innovations involve smart homes, help robots, e-textiles, and portable and implantable sensors. In the following sections, the proposed research methodology is discussed in more detail. Figure 2 shows a functional diagram of the research methodology.

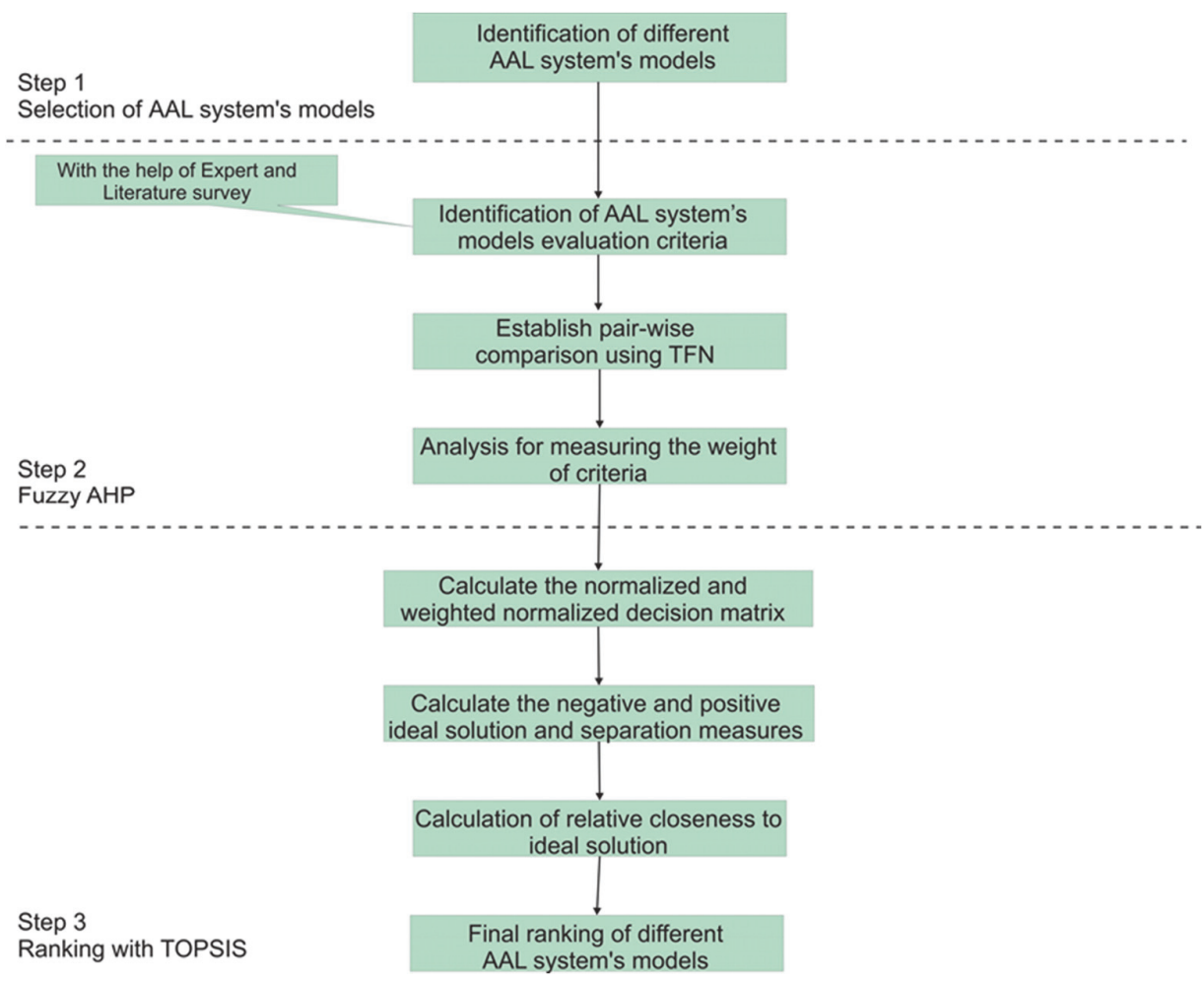

Figure 2. Functional diagram of the research methodology.

\subsection{Step 1: Identification of Different AAL System's Models}

A variety of ventures have adopted the AAL scope as a field of research. Various references, symmetrical models, systems, and interfaces have been proposed for an acceptable AAL scheme, but few of these have been generally accepted. In this section, we analyze the most comprehensive Ambient Assisted Living system's symmetrical models. A graphical representation of the hierarchy can be seen in Figure 3. 


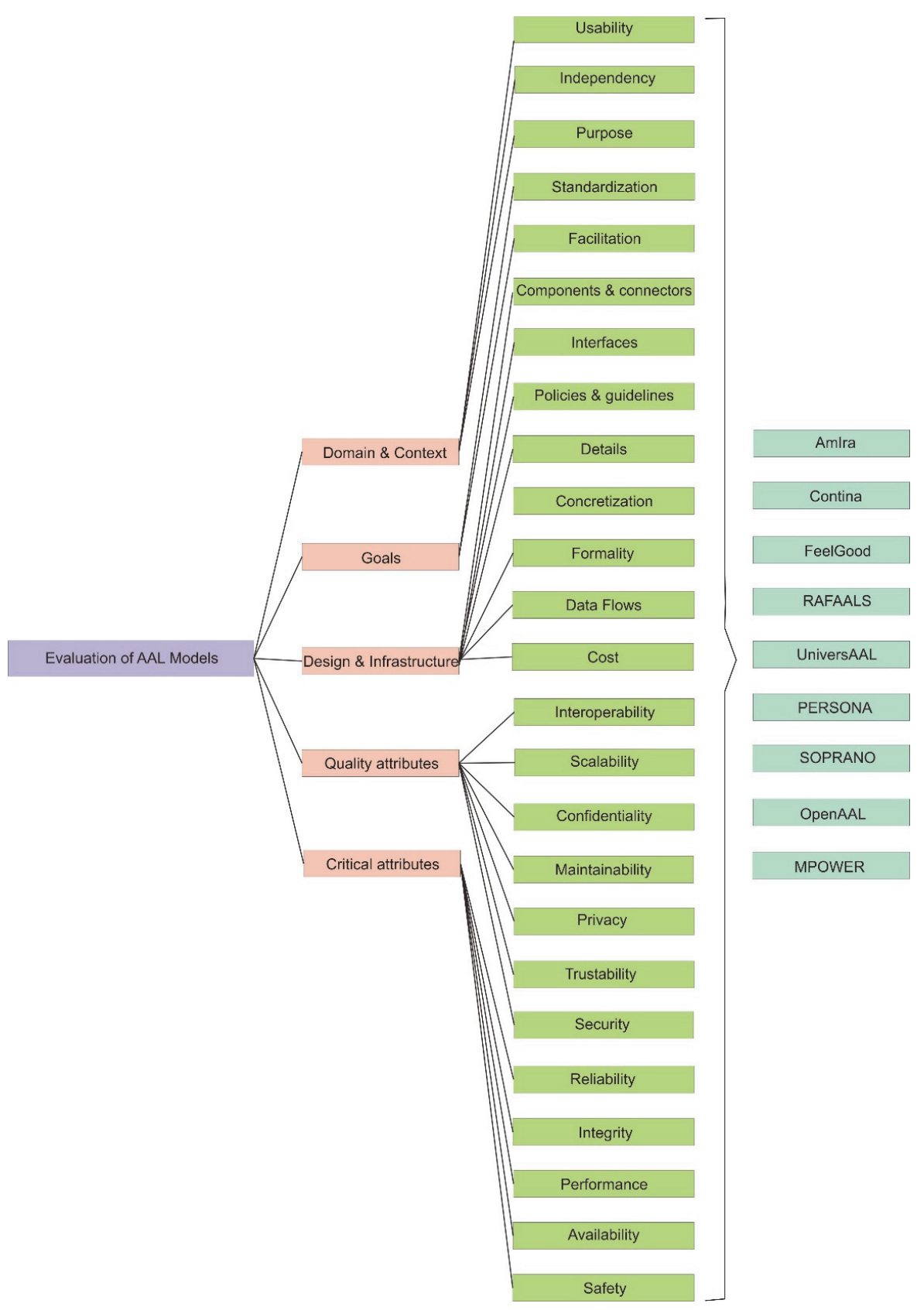

Figure 3. Structure for evaluation of different Ambient Assisted Living system's symmetrical models.

\section{A. Ambient Intelligence Reference Architecture (AmIRA)}

AmIRA includes an emphasis on processes, structures, and components [23]. It assimilates multi-layer application architecture. According to the given specifications of ambient intelligent systems, the intention of AmIRA is to encourage the re-use of Ambient Intelligence (AmI) processes throughout various AmI systems [24]. The advantage of this design is that each layer is autonomous; however, there is a demand for resources from other layers. AmIRA is uncertain because there are no restrictions mentioned, however, the framework components do not adequately define the business processes.

\section{B. Continua}

Continua is an attempt to provide compatibility in the area of personal telemedicine. The design identifies different steps and reference system classes [25]. Continua made an explicit option to build a framework that incorporates several domains. It is known to be the only approach that provides a uniform security system. Continua reflects the 
reference architecture mostly as a symmetrical layered model. Each link is composed to its counterparts by a platform that has been identified. Reference system classes and platforms are the maximum representation level in the Continua design. Conversely, it depends on the execution of the design and lacks any applications.

\section{FeelGood}

FeelGood is an initiative that introduces new architectural elements to complement existing practices by specifying the ecosystem level of service components [26]. The primary objective was to enhance the quality of life in Finland. The suggested RA does not describe comprehensive interfaces, but offers interface specifications and refers to the applicable requirements that could be implemented. The purpose of this RA was to direct the development of the product. Stakeholders and comprehensive services were identified.

\section{RAFAALS}

RAFAALS relates to the reference design for AAL systems $[27,28]$ as a SOA-based symmetrical model of AAL. The key architecture is based on the principle of separating the functionality and the configuration of the data flow among the layers of the process. It facilitates the transfer of events between flexible software applications wherein its modules allow use of little to no awareness of other elements. This structure distinguishes the activities within each evolved producer. The layers are analytically isolated from each other and clearly specified. This is a traditional structural platform that enables the design, formulation, and deployment of any AAL setting. It defines modules and connections in an abstract way, making them a comprehensive, functional, and systematic architecture.

\section{E. Univers $A A L$}

The UniversAAL system is an open platform designed to promote the development, delivery, and implementation of technology solutions towards assisted living environments. This system is utilized to encourage end-users (i.e., supported individuals, their parents, and communities), AAL-responsible authorities, and organizations involved in the creation and implementation of AAL services. It comprises of a wide variety of tools (some are applications and others are models/architectures) targeted at these various classes. Services are divided into three major groups: runtime assistance, production assistance, and community assistance. UniversAAL is regarded to be one of the most comprehensive RMs to date [29,30]. It represents an interpretation of AAL structures at the maximum level of abstraction [31], utilizing as few terms as possible. It also reflects the AAL domain description, the AAL space review, the types of technology included, and several other principles.

\section{F. PERSONA}

PERSONA is a service platform for AAL environments; it is designed to promote the incremental creation of AAL areas focused on a compact foundation. AAL spaces are modelled as accessible distributed systems in PERSONA. The system depends on the administrative re-configuration of the platform elements, including the Situation Reasoner, the Dialog Manager, and the Service Orchestra, to provide aggregated benefits [32].

\section{G. SOPRANO}

SOPRANO is a highly configurable, open AAL framework for senior citizens focused on semantic agreements. This serves as a facilitating artifact among the different modules of the process by establishing a uniform integrated terminology for various layers of abstraction. The architecture of SOPRANO offers exclusively predetermined contractbased interfaces for various stakeholders centered on structured ontology [33]. Ontology serves as a facilitating artifact between the modules of the different structure by providing a shared interconnected language for different levels of abstraction. The SOPRANO initiative has been an important guide for several other initiatives, such as OpenAAL [34], which acts as a comprehensive ontology for AAL symmetrical models. 


\section{H. OpenAAL}

OpenAAL is a collaborative open-source program of the FZI Research Center for Information Technologies, Friedrich-Schiller-University of Jena, and CAS Software AG. It provides a scalable and efficient interface for AAL situations and is focused on the research findings of many German and foreign initiatives, such as the SOPRANO2 Combined Project. The OpenAAL framework allows for easy deployment, configuration, and scenariodependent provision of versatile, context-aware, and customized IT-based functions [29].

\section{MPOWER}

MPOWER is an AAL initiative with the goal of creating and maintaining AAL frameworks based on trends, service-oriented frameworks, online services, and Extensible Stylesheet Language (XSDL) transformations [35]. It emphasizes compatibility among services, extensively in the areas of AAL.

\subsection{Step 2: Fuzzy AHP}

Saaty suggested the Analytic Hierarchy Process (AHP) method in 1990. All numerical and contextual considerations are integrated into AHP in the decision-making procedure. Because the highly classified scale of 1 to 9 is often used in the AHP process, this method is commonly criticized for not integrating uncertainty into the decision-making procedure. The fuzzy AHP approach has also been used in many fields to overcome multi-criteria challenges. Haq and Kannan [36] used this approach to pick the best supplier in the supply chain. This was also used by Huang et al. [37] for the evaluation of R\&D projects. Fuzzy set theory is essentially a type of classical set theory. It is based on an adjacency matrix, and assigns a rank between one and ten. If the symbol is a fuzzy package, a tilde (i.e., $\sim$ ) is placed over it. A fuzzy activity is defined by $(\mathrm{l}, \mathrm{m}, \mathrm{u})$ whereby ' $\mathrm{l}$ ' is the lowest number, ' $\mathrm{m}$ ' is the most probable value, and ' $u$ ' is the maximum priority [19].

\subsection{Step 3: Ranking with TOPSIS}

On the basis of the results, alternatives are rated using TOPSIS. In this process, the following types of parameters or characteristics are regarded:

- Domain and context

- Goals

- Design and infrastructure

- Quality attributes

- Critical attributes

In this analysis, different kinds of alternatives are evaluated as follows:

- Negative ideal solution

- Positive ideal solution

TOPSIS is focused on the choice of the most appropriate alternative or initiative that is the furthest from the negative ideal solution and the nearest to the positive ideal solution. The positive and negative ideal solutions are those with the maximum and minimum benefits, respectively.

\section{Results}

This section addresses the various quantitative measurements of the integrated fuzzy deployment of the AHP-TOPSIS symmetrical method. To achieve this goal, in our research study, we used the combined fuzzy AHP-TOPSIS approach, a well-established and verified decision-making technique. This methodology is designed to evaluate different Ambient Assisted Living system's symmetrical models based on their impact assessment in the current information technology era. To create a more compelling result, we took recommendations from 79 experts with diverse technologies and academic abilities.

To evaluate the different Ambient Assisted Living system's symmetrical models from a user perspective, five Level-1 parameters, namely domain and context, goals, design and 
infrastructure, quality attributes, and critical attributes, were defined respectively as LC1, LC2, LC3, LC4 and LC5. Further sub-parameters for domain and context were usability, independency, and purpose, defined respectively as LC11, LC12 and LC13, LC14 and LC5. Goals sub-parameters were standardization and facilitation, defined respectively as LC21 and LC22. Sub-parameters for design and infrastructure were components and connections, interfaces, policies and guidelines, details, concretization, formality, data flows, and cost, defined respectively as LC31, LC32, LC33, LC34, LC35, LC36, LC37 and LC38. Quality attributes sub-parameters were interoperability, scalability, confidentiality, maintainability, privacy, trustability, and security, defined respectively as LC41, LC42, LC43, LC44, LC45, LC46 and LC47. Critical attributes sub-parameters were reliability, integrity, performance, availability, and safety, defined respectively as LC51, LC52, LC53, LC54 and LC55. Different alternatives to the Ambient Assisted Living system's symmetrical model were AmIRA, Continua, FeelGood, RAFAALS, UniversAAL, PERSONA, SOPRANO, OpenAAL, and MPOWER denoted by AT1, AT2, AT3, AT4, AT5, AT6, AT7, AT8 and AT9 respectively. The local criteria and sub-criteria weights were calculated using pair-wise comparison matrices.

The pair-wise comparative matrix for the level 1 factor was created, as shown in Table 1. The compound pair-wise relative matrixes for the hierarchical diagram of level 2 are also specified in Tables 2-6. Table 7 shows the defuzzification matrix with alpha cut method and local weights. Tables $8-12$ show aggregated pair-wise comparison matrixes at level 2 for domain and context, goals, design and infrastructure, quality attributes, and critical attributes, respectively. To be more specific, integration was executed to quantify the element weights of each point. In addition, with the support of the hierarchical structure, Table 13 and Figure 4 represent the overall weights and ranking of methods.

Table 1. Fuzzy pair-wise comparison matrix at level 1.

\begin{tabular}{|c|c|c|c|c|c|}
\hline Level 1 & LC1 & LC2 & LC3 & LC4 & LC5 \\
\hline LC1 & $\begin{array}{l}1.00000 \\
1.00000 \\
1.00000\end{array}$ & $\begin{array}{l}1.87222, \\
2.52710 \\
3.20315\end{array}$ & $\begin{array}{l}1.46140 \\
1.68142, \\
1.97431\end{array}$ & $\begin{array}{l}1.44161, \\
2.43185, \\
3.38615\end{array}$ & $\begin{array}{l}0.46177, \\
0.57214, \\
0.78451\end{array}$ \\
\hline LC2 & - & $\begin{array}{l}1.00000 \\
1.00000 \\
1.00000\end{array}$ & $\begin{array}{l}0.60183 \\
0.77154 \\
1.02165\end{array}$ & $\begin{array}{c}0.77108 \\
0.9504 \\
1.21361\end{array}$ & $\begin{array}{c}0.16130, \\
0.19513, \\
0.24917\end{array}$ \\
\hline LC3 & - & - & $\begin{array}{l}1.00000 \\
1.00000 \\
1.00000\end{array}$ & $\begin{array}{l}0.71694, \\
1.01502, \\
1.35153\end{array}$ & $\begin{array}{l}0.20186, \\
0.24162, \\
0.31117\end{array}$ \\
\hline $\mathrm{LC} 4$ & - & - & - & $\begin{array}{l}1.00000 \\
1.00000 \\
1.00000\end{array}$ & $\begin{array}{l}0.19516, \\
0.22813 \\
0.21903\end{array}$ \\
\hline LC5 & - & - & - & - & $\begin{array}{l}1.00000 \\
1.00000 \\
1.00000\end{array}$ \\
\hline
\end{tabular}

Table 2. Fuzzy aggregated pair-wise comparison matrix at level 2 for domain and context.

\begin{tabular}{cccc}
\hline Level 2 for $\mathbf{1}$ & LC11 & LC12 & LC13 \\
\hline LC11 & $1.00000,1.00000,1.00000$ & $0.68918,0.88160,1.10012$ & $0.22515,0.27612,0.35714$ \\
LC12 & - & $1.00000,1.00000,1.00000$ & $0.30151,0.38912,0.56091$ \\
LC13 & - & - & $1.00000,1.00000,1.00000$ \\
\hline
\end{tabular}

Table 3. Fuzzy aggregated pair-wise comparison matrix at level 2 for goals.

\begin{tabular}{ccc}
\hline Level $\mathbf{2}$ for $\mathbf{2}$ & LC21 & LC22 \\
\hline LC21 & $1.00000,1.00000,1.00000$ & $0.65751,1.16531,1.68831$ \\
LC22 & - & $1.00000,1.00000,1.00000$ \\
\hline
\end{tabular}


Table 4. Fuzzy aggregated pair-wise comparison matrix at level 2 for design and infrastructure.

\begin{tabular}{|c|c|c|c|c|c|c|c|c|}
\hline & LC31 & LC32 & LC33 & LC34 & LC35 & LC36 & LC37 & LC38 \\
\hline LC31 & $\begin{array}{l}1.00000 \\
1.00000 \\
1.00000\end{array}$ & $\begin{array}{l}1.00100 \\
1.51157 \\
1.93311\end{array}$ & $\begin{array}{l}0.48916 \\
0.63712, \\
1.00010\end{array}$ & $\begin{array}{l}0.41152, \\
0.57413, \\
1.00001\end{array}$ & $\begin{array}{l}0.22115 \\
0.28171 \\
0.41152\end{array}$ & $\begin{array}{l}0.31146 \\
0.46110 \\
0.87015\end{array}$ & $\begin{array}{l}0.65175 \\
1.16513, \\
1.68813\end{array}$ & $\begin{array}{c}0.24144, \\
0.32318, \\
0.48011\end{array}$ \\
\hline LC32 & - & $\begin{array}{l}1.00000 \\
1.00000 \\
1.00000\end{array}$ & $\begin{array}{l}0.57413 \\
0.66517 \\
0.80221\end{array}$ & $\begin{array}{l}0.30319, \\
0.39316, \\
0.56611\end{array}$ & $\begin{array}{l}0.26719 \\
0.35211 \\
0.51716\end{array}$ & $\begin{array}{l}0.16613, \\
0.19619, \\
0.25311\end{array}$ & $\begin{array}{l}0.39310, \\
0.57413, \\
1.05614\end{array}$ & $\begin{array}{c}0.16912, \\
0.20716, \\
0.27519\end{array}$ \\
\hline LC33 & - & - & $\begin{array}{l}1.00000 \\
1.00000 \\
1.00000\end{array}$ & $\begin{array}{l}1.0000 \\
1.3195 \\
1.5518\end{array}$ & $\begin{array}{l}0.3009 \\
0.4352 \\
0.8027\end{array}$ & $\begin{array}{l}0.80127, \\
0.87015, \\
1.00010\end{array}$ & $\begin{array}{l}1.26119 \\
1.82510 \\
2.43314\end{array}$ & $\begin{array}{l}0.17218, \\
0.20911, \\
0.26481\end{array}$ \\
\hline LC34 & - & - & - & $\begin{array}{l}1.00000 \\
1.00000 \\
1.00000\end{array}$ & $\begin{array}{l}0.53186, \\
0.91143, \\
1.58316\end{array}$ & $\begin{array}{l}0.60813, \\
1.05192, \\
1.68219\end{array}$ & $\begin{array}{l}0.75103, \\
1.34165, \\
1.96111\end{array}$ & $\begin{array}{l}0.67910, \\
0.74819, \\
0.87105\end{array}$ \\
\hline LC35 & - & - & - & - & $\begin{array}{l}1.00000 \\
1.00000 \\
1.00000\end{array}$ & $\begin{array}{l}0.41152, \\
0.63712, \\
1.17191\end{array}$ & $\begin{array}{l}0.94165, \\
1.10195 \\
1.24157\end{array}$ & $\begin{array}{l}0.25100 \\
0.33100 \\
0.50100\end{array}$ \\
\hline LC36 & - & - & - & - & - & $\begin{array}{l}1.00000 \\
1.00000 \\
1.00000\end{array}$ & $\begin{array}{l}1.88181 \\
2.55108 \\
3.16197\end{array}$ & $\begin{array}{l}0.80127, \\
1.03152, \\
1.31160\end{array}$ \\
\hline LC37 & - & - & - & - & - & - & $\begin{array}{l}1.00000 \\
1.00000 \\
1.00000\end{array}$ & $\begin{array}{c}0.21136, \\
0.21575, \\
0.31195\end{array}$ \\
\hline LC38 & - & - & - & - & - & - & - & $\begin{array}{l}1.00000 \\
1.00000 \\
1.00000\end{array}$ \\
\hline
\end{tabular}

Table 5. Fuzzy aggregated pair-wise comparison matrix at level 2 for quality attributes.

\begin{tabular}{|c|c|c|c|c|c|c|c|}
\hline & LC41 & LC42 & LC43 & LC44 & LC45 & LC46 & LC47 \\
\hline LC41 & $\begin{array}{l}1.00000 \\
1.00000 \\
1.00000\end{array}$ & $\begin{array}{l}0.31127, \\
0.43195, \\
0.62152\end{array}$ & $\begin{array}{l}0.87313, \\
0.90112 \\
0.94165\end{array}$ & $\begin{array}{l}0.22161 \\
0.29128 \\
0.41166\end{array}$ & $\begin{array}{l}0.16163, \\
0.19169, \\
0.21531\end{array}$ & $\begin{array}{l}0.22161 \\
0.29128 \\
0.41166\end{array}$ & $\begin{array}{l}0.16163, \\
0.19169, \\
0.25311\end{array}$ \\
\hline LC42 & - & $\begin{array}{l}1.00000 \\
1.00000 \\
1.00000\end{array}$ & $\begin{array}{l}0.5743 \\
0.6657 \\
0.8022\end{array}$ & $\begin{array}{l}0.3039 \\
0.3936 \\
0.5661\end{array}$ & $\begin{array}{l}0.8027 \\
0.8705 \\
1.0000\end{array}$ & $\begin{array}{l}0.9465 \\
1.1095 \\
1.2457\end{array}$ & $\begin{array}{l}1.26119, \\
1.82510, \\
2.43314\end{array}$ \\
\hline LC43 & - & - & $\begin{array}{l}1.00000 \\
1.00000 \\
1.00000\end{array}$ & $\begin{array}{l}1.00010, \\
1.31915, \\
1.55118\end{array}$ & $\begin{array}{l}0.60813, \\
1.05912, \\
1.68219\end{array}$ & $\begin{array}{l}1.88811 \\
2.55018 \\
3.16917\end{array}$ & $\begin{array}{l}0.75103, \\
1.34165, \\
1.96111\end{array}$ \\
\hline LC44 & - & - & - & $\begin{array}{l}1.00000 \\
1.00000 \\
1.00000\end{array}$ & $\begin{array}{l}0.53816 \\
0.91413 \\
1.58316\end{array}$ & $\begin{array}{l}0.60813, \\
1.05912, \\
1.68219\end{array}$ & $\begin{array}{l}0.75013, \\
1.34615, \\
1.96111\end{array}$ \\
\hline LC45 & - & - & - & - & $\begin{array}{l}1.00000 \\
1.00000 \\
1.00000\end{array}$ & $\begin{array}{l}0.41152, \\
0.63172, \\
1.17191\end{array}$ & $\begin{array}{l}0.94615, \\
1.10915, \\
1.24517\end{array}$ \\
\hline LC46 & - & - & - & - & - & $\begin{array}{l}1.00000 \\
1.00000 \\
1.00000\end{array}$ & $\begin{array}{l}1.88181, \\
2.55108, \\
3.16197\end{array}$ \\
\hline LC47 & - & - & - & - & - & - & $\begin{array}{l}1.00000, \\
1.00000, \\
1.00000\end{array}$ \\
\hline
\end{tabular}


Table 6. Fuzzy aggregated pair-wise comparison matrix at level 2 for critical attributes.

\begin{tabular}{|c|c|c|c|c|c|}
\hline & LC51 & LC52 & LC53 & LC54 & LC55 \\
\hline LC51 & $\begin{array}{l}1.00000 \\
1.00000 \\
1.00000\end{array}$ & $\begin{array}{l}0.97110, \\
1.24175, \\
1.60194\end{array}$ & $\begin{array}{l}1.05912, \\
1.58419, \\
2.22016\end{array}$ & $\begin{array}{l}0.77313, \\
1.01118, \\
1.28181\end{array}$ & $\begin{array}{l}0.761112, \\
0.912110, \\
1.096115\end{array}$ \\
\hline LC52 & - & $\begin{array}{l}1.00000 \\
1.00000 \\
1.00000\end{array}$ & $\begin{array}{l}0.63512, \\
0.91143, \\
1.34310\end{array}$ & $\begin{array}{l}0.42713, \\
0.63315 \\
0.96610\end{array}$ & $\begin{array}{l}0.34716, \\
0.49010, \\
0.87314\end{array}$ \\
\hline LC53 & - & - & $\begin{array}{l}1.00000 \\
1.00000 \\
1.00000\end{array}$ & $\begin{array}{l}0.51416, \\
0.65715, \\
0.78146\end{array}$ & $\begin{array}{l}0.52113, \\
0.65917, \\
0.91191\end{array}$ \\
\hline LC54 & - & - & - & $\begin{array}{l}1.00000 \\
1.00000 \\
1.00000\end{array}$ & $\begin{array}{l}0.55612, \\
0.64148 \\
0.81122\end{array}$ \\
\hline LC55 & - & - & - & - & $\begin{array}{l}1.00000 \\
1.00000 \\
1.00000\end{array}$ \\
\hline
\end{tabular}

Table 7. Defuzzification matrix with alpha cut method and local weights.

\begin{tabular}{ccccccc}
\hline Level 1 & LC1 & LC2 & LC3 & LC4 & LC5 & Weights \\
\hline LC1 & 1.00000 & 2.55144 & 1.71017 & 2.42174 & 0.59193 & 0.240000 \\
\hline LC2 & 0.39115 & 1.00000 & 0.79164 & 0.97169 & 0.20173 & 0.095200 \\
\hline LC3 & 0.58176 & 1.25516 & 1.00000 & 1.05163 & 0.25132 & 0.120000 \\
\hline LC4 & 0.41120 & 1.02136 & 0.94167 & 1.00000 & 0.23157 & 0.103200 \\
\hline LC5 & 1.66186 & 4.82139 & 3.94195 & 4.21427 & 1.00000 & 0.441600 \\
\hline \multicolumn{7}{c}{ CR $=0.00250254$} \\
\end{tabular}

Table 8. Aggregated pair-wise comparison matrix at level 2 for domain and context.

\begin{tabular}{ccccc}
\hline Level 2 for 1 & LC11 & LC12 & LC13 & Weights \\
\hline LC11 & 1.00000 & 0.81905 & 0.28139 & 0.183200 \\
\hline LC12 & 1.12130 & 1.00000 & 0.41111 & 0.223900 \\
\hline LC13 & 3.52124 & 2.43125 & 1.00000 & 0.592900 \\
\hline
\end{tabular}

Table 9. Aggregated pair-wise comparison matrix at level 2 for goals.

\begin{tabular}{cccc}
\hline Level $\mathbf{2}$ for $\mathbf{2}$ & LC21 & LC22 & Weights \\
\hline LC21 & 1.00000 & 1.16911 & 0.5391000 \\
\hline LC22 & 0.85154 & 1.00000 & 0.4611000 \\
\hline \multicolumn{3}{c}{ C.R. $=0.001$} \\
\hline
\end{tabular}


Table 10. Aggregated pair-wise comparison matrix at level 2 for design and infrastructure.

\begin{tabular}{cccccccccc}
\hline & LC31 & LC32 & LC33 & LC34 & LC35 & LC36 & LC37 & LC38 & Weights \\
\hline LC31 & 1.00000 & 1.409012 & 0.69001 & 0.60410 & 0.30002 & 0.52060 & 1.10069 & 0.34300 & 0.073300 \\
\hline LC32 & 0.67006 & 1.00000 & 0.67700 & 0.40143 & 0.37204 & 0.20033 & 0.64095 & 0.21051 & 0.049700 \\
\hline LC33 & 1.44070 & 1.47071 & 1.00000 & 1.29077 & 0.49305 & 0.85200 & 1.83064 & 0.21040 & 0.103100 \\
\hline LC34 & 1.56000 & 2.41307 & 0.77006 & 1.00000 & 0.96036 & 1.10024 & 1.35011 & 0.73190 & 0.127100 \\
\hline LC35 & 3.30306 & 2.68503 & 2.02603 & 1.03078 & 1.00000 & 0.71072 & 1.10028 & 0.43500 & 0.141400 \\
\hline LC36 & 1.89802 & 4.91808 & 1.17307 & 0.90071 & 1.39043 & 1.00000 & 2.38052 & 1.00473 & 0.172900 \\
\hline LC37 & 0.85540 & 1.53907 & 0.54405 & 0.74001 & 0.90679 & 0.41902 & 1.00000 & 0.26021 & 0.076000 \\
\hline LC38 & 2.91054 & 4.64900 & 4.67029 & 1.36631 & 2.29089 & 0.95048 & 3.81053 & 1.00000 & 0.256500 \\
\hline
\end{tabular}

Table 11. Aggregated pair-wise comparison matrix at level 2 for quality attributes.

\begin{tabular}{ccccccccc}
\hline & LC41 & LC42 & LC43 & LC44 & LC45 & LC46 & LC47 & Weights \\
\hline LC41 & 1.00000 & 1.4912 & 0.69010 & 0.64010 & 0.30207 & 0.52608 & 1.16901 & 0.098700 \\
\hline LC42 & 0.67006 & 1.00000 & 0.6770 & 0.41403 & 0.37024 & 0.20033 & 0.64905 & 0.120700 \\
\hline LC43 & 1.44700 & 1.47701 & 1.00000 & 1.29077 & 0.49305 & 0.85200 & 1.83064 & 0.119600 \\
\hline LC44 & 1.56000 & 2.41370 & 0.77006 & 1.00000 & 0.96306 & 1.10204 & 1.35101 & 0.166500 \\
\hline LC45 & 3.30360 & 2.68503 & 2.02063 & 1.03708 & 1.00000 & 0.71702 & 1.10028 & 0.178500 \\
\hline LC46 & 1.89082 & 4.91088 & 1.17037 & 0.90071 & 1.39043 & 1.00000 & 2.38052 & 0.116000 \\
\hline LC47 & 0.85054 & 1.53907 & 0.54045 & 0.74001 & 0.90679 & 0.41925 & 1.00000 & 0.200000 \\
\hline \multicolumn{7}{c}{ C.R. $=0.03548$} \\
\hline
\end{tabular}

Table 12. Aggregated pair-wise comparison matrix at level 2 for critical attributes.

\begin{tabular}{ccccccc}
\hline & LC51 & LC52 & LC53 & LC54 & LC55 & Weights \\
\hline LC51 & 1.00000 & 1.26890 & 1.61240 & 1.02103 & 0.92004 & 0.221600 \\
\hline LC52 & 0.78081 & 1.00000 & 1.26903 & 0.66501 & 0.55003 & 0.159600 \\
\hline LC53 & 0.62002 & 0.78708 & 1.00000 & 0.65306 & 0.69000 & 0.144600 \\
\hline LC54 & 0.97901 & 1.50305 & 1.53000 & 1.00000 & 0.66405 & 0.211500 \\
\hline LC55 & 1.08605 & 1.81702 & 1.44903 & 1.50409 & 1.00000 & 0.262700 \\
\hline \multicolumn{7}{c}{ C.R. $=0.0069$} \\
\hline
\end{tabular}

Table 13. Overall weights and ranking of methods.

\begin{tabular}{cccccc}
\hline Level 1 & Local Weights & Leve1 2 & Local Weights & Global Weights & Normalized Weights \\
\hline \multirow{2}{*}{ LC1 } & \multirow{2}{*}{0.240000} & LC11 & 0.183200 & 0.043968 & 0.045086 \\
\cline { 3 - 6 } & & LC12 & 0.223900 & 0.053736 & 0.055103 \\
\cline { 3 - 6 } LC2 & \multirow{2}{*}{0.095200} & LC13 & 0.592900 & 0.142296 & 0.145915 \\
\cline { 3 - 6 } & & LC21 & 0.539000 & 0.051313 & 0.052618 \\
\hline
\end{tabular}


Table 13. Cont.

\begin{tabular}{|c|c|c|c|c|c|}
\hline Level 1 & Local Weights & Level 2 & Local Weights & Global Weights & Normalized Weights \\
\hline \multirow{8}{*}{ LC3 } & \multirow{8}{*}{0.120000} & LC31 & 0.073300 & 0.006978 & 0.007155 \\
\hline & & LC32 & 0.049700 & 0.004732 & 0.004852 \\
\hline & & LC33 & 0.103100 & 0.009815 & 0.010065 \\
\hline & & LC34 & 0.127100 & 0.012099 & 0.012407 \\
\hline & & LC35 & 0.141400 & 0.013461 & 0.013803 \\
\hline & & LC36 & 0.172900 & 0.016460 & 0.016879 \\
\hline & & LC37 & 0.076000 & 0.007235 & 0.007419 \\
\hline & & LC38 & 0.256500 & 0.024419 & 0.02504 \\
\hline \multirow{7}{*}{$\mathrm{LC} 4$} & \multirow{7}{*}{0.103200} & LC41 & 0.098700 & 0.010186 & 0.010445 \\
\hline & & LC42 & 0.120700 & 0.012456 & 0.012773 \\
\hline & & LC43 & 0.119600 & 0.012343 & 0.012657 \\
\hline & & LC44 & 0.166500 & 0.017183 & 0.01762 \\
\hline & & LC45 & 0.178500 & 0.018421 & 0.018889 \\
\hline & & LC46 & 0.116000 & 0.011971 & 0.012275 \\
\hline & & LC47 & 0.200000 & 0.020640 & 0.021165 \\
\hline \multirow{5}{*}{ LC5 } & \multirow{5}{*}{0.441600} & LC51 & 0.221600 & 0.097859 & 0.100348 \\
\hline & & LC52 & 0.159600 & 0.070479 & 0.072271 \\
\hline & & LC53 & 0.144600 & 0.063855 & 0.065479 \\
\hline & & LC54 & 0.211500 & 0.093398 & 0.095773 \\
\hline & & LC55 & 0.262700 & 0.116008 & 0.118958 \\
\hline
\end{tabular}

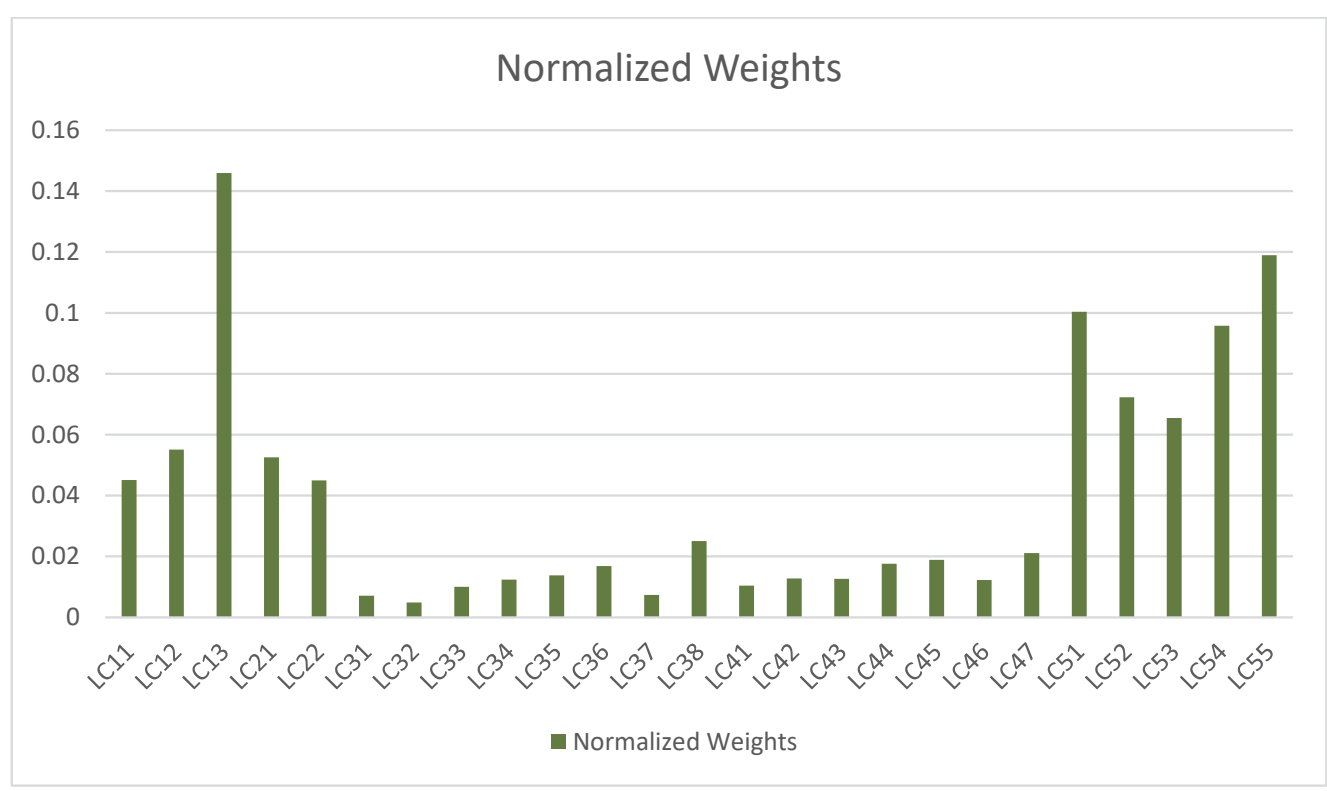

Figure 4. Graphical representation of overall weights.

Table 14 shows the subjective cognition results of evaluators in linguistic terms. Table 15 presents the normalized fuzzy decision matrix. Table 16 presents the weighted normalized fuzzy decision matrix. Finally, Table 17 and Figure 5 shows the closeness coefficients to the aspired level among the different alternatives. 
Table 14. Subjective cognition results of evaluators in linguistic terms.

\begin{tabular}{|c|c|c|c|c|c|c|c|c|c|}
\hline & AT1 & AT2 & AT3 & AT4 & AT5 & AT6 & AT7 & AT8 & AT9 \\
\hline LC11 & $\begin{array}{l}5.120 \\
7.140 \\
8.720\end{array}$ & $\begin{array}{l}3.150 \\
5.150 \\
6.910\end{array}$ & $\begin{array}{l}2.820 \\
4.640 \\
6.640\end{array}$ & $\begin{array}{l}1.550 \\
3.180 \\
5.180\end{array}$ & $\begin{array}{l}5.120 \\
7.140, \\
8.720\end{array}$ & $\begin{array}{l}3.150 \\
5.150 \\
6.910\end{array}$ & $\begin{array}{l}2.820 \\
4.640 \\
6.640\end{array}$ & $\begin{array}{l}1.550 \\
3.180 \\
5.180\end{array}$ & $\begin{array}{l}1.450 \\
3.180 \\
5.180\end{array}$ \\
\hline LC12 & $\begin{array}{l}4.280 \\
6.370 \\
8.370\end{array}$ & $\begin{array}{l}2.820 \\
4.640 \\
6.640\end{array}$ & $\begin{array}{l}1.550 \\
3.180 \\
5.180\end{array}$ & $\begin{array}{l}5.120 \\
7.140 \\
8.720\end{array}$ & $\begin{array}{l}3.150 \\
5.150 \\
6.910\end{array}$ & $\begin{array}{l}2.450 \\
4.450 \\
6.450\end{array}$ & $\begin{array}{l}2.910 \\
4.640 \\
6.550\end{array}$ & $\begin{array}{l}1.450 \\
3.000 \\
4.910\end{array}$ & $\begin{array}{l}1.180 \\
2.820 \\
4.820\end{array}$ \\
\hline LC13 & $\begin{array}{l}4.270 \\
6.270 \\
8.140\end{array}$ & $\begin{array}{l}2.910 \\
4.640 \\
6.550\end{array}$ & $\begin{array}{l}1.450 \\
3.000 \\
4.910\end{array}$ & $\begin{array}{l}4.280 \\
6.370 \\
8.370\end{array}$ & $\begin{array}{l}2.820 \\
4.640 \\
6.640\end{array}$ & $\begin{array}{l}1.550 \\
3.180 \\
5.180\end{array}$ & $\begin{array}{l}5.120 \\
7.140 \\
8.720\end{array}$ & $\begin{array}{l}3.150 \\
5.150 \\
6.910\end{array}$ & $\begin{array}{l}2.450 \\
4.450 \\
6.450\end{array}$ \\
\hline LC21 & $\begin{array}{l}5.360 \\
7.360 \\
9.120\end{array}$ & $\begin{array}{l}5.120 \\
7.140 \\
8.720\end{array}$ & $\begin{array}{l}3.150 \\
5.150 \\
6.910\end{array}$ & $\begin{array}{l}2.820 \\
4.640 \\
6.640\end{array}$ & $\begin{array}{l}2.910 \\
4.640 \\
6.550\end{array}$ & $\begin{array}{l}1.450 \\
3.000 \\
4.910\end{array}$ & $\begin{array}{l}4.280 \\
6.370 \\
8.370\end{array}$ & $\begin{array}{l}2.450 \\
4.450 \\
6.450\end{array}$ & $\begin{array}{l}2.820 \\
4.640 \\
6.640\end{array}$ \\
\hline LC22 & $\begin{array}{l}4.640 \\
6.640 \\
8.550\end{array}$ & $\begin{array}{l}2.820 \\
4.640 \\
6.640\end{array}$ & $\begin{array}{l}1.550 \\
3.180 \\
5.180\end{array}$ & $\begin{array}{l}5.120 \\
7.140 \\
8.720\end{array}$ & $\begin{array}{l}3.150 \\
5.150 \\
6.910\end{array}$ & $\begin{array}{l}3.150 \\
5.150 \\
6.910\end{array}$ & $\begin{array}{l}2.820 \\
4.640 \\
6.640\end{array}$ & $\begin{array}{l}5.360 \\
7.360 \\
9.120\end{array}$ & $\begin{array}{l}2.450 \\
4.450 \\
6.450\end{array}$ \\
\hline LC31 & $\begin{array}{l}3.120 \\
5.000 \\
7.140\end{array}$ & $\begin{array}{l}2.910 \\
4.640 \\
6.550\end{array}$ & $\begin{array}{l}1.450 \\
3.000 \\
4.910\end{array}$ & $\begin{array}{l}4.280 \\
6.370 \\
8.370\end{array}$ & $\begin{array}{l}2.450 \\
4.450 \\
6.450\end{array}$ & $\begin{array}{l}2.450 \\
4.450 \\
6.450\end{array}$ & $\begin{array}{l}2.910 \\
4.640 \\
6.550\end{array}$ & $\begin{array}{l}4.640 \\
6.640 \\
8.550\end{array}$ & $\begin{array}{l}2.450 \\
4.450 \\
6.450\end{array}$ \\
\hline LC32 & $\begin{array}{l}4.280 \\
6.370 \\
8.370\end{array}$ & $\begin{array}{l}5.120 \\
7.140 \\
8.720\end{array}$ & $\begin{array}{l}3.150 \\
5.150 \\
6.910\end{array}$ & $\begin{array}{l}2.820 \\
4.640 \\
6.640\end{array}$ & $\begin{array}{l}5.360 \\
7.360 \\
9.120\end{array}$ & $\begin{array}{l}2.820 \\
4.640 \\
6.640\end{array}$ & $\begin{array}{l}2.820 \\
4.640 \\
6.640\end{array}$ & $\begin{array}{l}5.360 \\
7.360 \\
9.120\end{array}$ & $\begin{array}{l}1.180 \\
2.820 \\
4.820\end{array}$ \\
\hline LC33 & $\begin{array}{l}4.280 \\
6.370 \\
8.370\end{array}$ & $\begin{array}{l}4.280 \\
6.370 \\
8.370\end{array}$ & $\begin{array}{l}2.450 \\
4.450 \\
6.450\end{array}$ & $\begin{array}{l}2.910 \\
4.640 \\
6.550\end{array}$ & $\begin{array}{l}4.640 \\
6.640 \\
8.550\end{array}$ & $\begin{array}{l}1.820 \\
3.730 \\
5.730\end{array}$ & $\begin{array}{l}2.820 \\
4.640 \\
6.640\end{array}$ & $\begin{array}{l}5.360 \\
7.360 \\
9.120\end{array}$ & $\begin{array}{l}1.180, \\
2.820, \\
4.820\end{array}$ \\
\hline LC34 & $\begin{array}{l}4.270 \\
6.270 \\
8.140\end{array}$ & $\begin{array}{l}2.180 \\
4.090 \\
6.140\end{array}$ & $\begin{array}{l}2.820 \\
4.640 \\
6.640\end{array}$ & $\begin{array}{l}2.820 \\
4.640 \\
6.640\end{array}$ & $\begin{array}{l}5.360 \\
7.360 \\
9.120\end{array}$ & $\begin{array}{l}1.450 \\
3.000 \\
4.910\end{array}$ & $\begin{array}{l}4.280 \\
6.370 \\
8.370\end{array}$ & $\begin{array}{l}2.450 \\
4.450 \\
6.450\end{array}$ & $\begin{array}{l}2.450 \\
4.450 \\
6.450\end{array}$ \\
\hline LC35 & $\begin{array}{l}4.280 \\
6.370 \\
8.370\end{array}$ & $\begin{array}{l}3.550 \\
5.550 \\
7.450\end{array}$ & $\begin{array}{l}1.820 \\
3.730 \\
5.730\end{array}$ & $\begin{array}{l}2.820 \\
4.640 \\
6.640\end{array}$ & $\begin{array}{l}5.360 \\
7.360 \\
9.120\end{array}$ & $\begin{array}{l}1.450 \\
3.000 \\
4.910\end{array}$ & $\begin{array}{l}4.280 \\
6.370 \\
8.370\end{array}$ & $\begin{array}{l}2.450 \\
4.450 \\
6.450\end{array}$ & $\begin{array}{l}1.180, \\
2.820, \\
4.820\end{array}$ \\
\hline LC36 & $\begin{array}{l}4.270 \\
6.270 \\
8.140\end{array}$ & $\begin{array}{l}2.910 \\
4.640 \\
6.550\end{array}$ & $\begin{array}{l}1.450, \\
3.000, \\
4.910\end{array}$ & $\begin{array}{l}4.280 \\
6.370 \\
8.370\end{array}$ & $\begin{array}{l}2.450 \\
4.450 \\
6.450\end{array}$ & $\begin{array}{l}3.150 \\
5.150 \\
6.910\end{array}$ & $\begin{array}{l}2.820 \\
4.640 \\
6.640\end{array}$ & $\begin{array}{l}5.360 \\
7.360 \\
9.120\end{array}$ & $\begin{array}{l}2.450 \\
4.450 \\
6.450\end{array}$ \\
\hline LC37 & $\begin{array}{l}5.360 \\
7.360 \\
9.120\end{array}$ & $\begin{array}{l}2.910 \\
4.640 \\
6.550\end{array}$ & $\begin{array}{l}1.450 \\
3.000 \\
4.910\end{array}$ & $\begin{array}{l}4.280 \\
6.370 \\
8.370\end{array}$ & $\begin{array}{l}2.450 \\
4.450 \\
6.450\end{array}$ & $\begin{array}{l}1.450 \\
3.000 \\
4.910\end{array}$ & $\begin{array}{l}4.280 \\
6.370 \\
8.370\end{array}$ & $\begin{array}{l}2.450 \\
4.450 \\
6.450\end{array}$ & $\begin{array}{l}2.820 \\
4.640, \\
6.640\end{array}$ \\
\hline LC38 & $\begin{array}{l}4.280 \\
6.370 \\
8.370\end{array}$ & $\begin{array}{l}5.120 \\
7.140 \\
8.720 \\
\end{array}$ & $\begin{array}{l}3.150 \\
5.150 \\
6.910\end{array}$ & $\begin{array}{l}2.820 \\
4.640 \\
6.640\end{array}$ & $\begin{array}{l}5.360 \\
7.360 \\
9.120\end{array}$ & $\begin{array}{l}3.150 \\
5.150 \\
6.910\end{array}$ & $\begin{array}{l}2.820 \\
4.640 \\
6.640\end{array}$ & $\begin{array}{l}5.360 \\
7.360 \\
9.120\end{array}$ & $\begin{array}{l}1.180 \\
2.820 \\
4.820\end{array}$ \\
\hline LC41 & $\begin{array}{l}4.270 \\
6.270 \\
8.140\end{array}$ & $\begin{array}{l}2.910 \\
4.640 \\
6.550\end{array}$ & $\begin{array}{l}1.450 \\
3.000 \\
4.910\end{array}$ & $\begin{array}{l}4.280 \\
6.370 \\
8.370\end{array}$ & $\begin{array}{l}2.450 \\
4.450 \\
6.450\end{array}$ & $\begin{array}{l}2.450 \\
4.450 \\
6.450\end{array}$ & $\begin{array}{l}2.910 \\
4.640 \\
6.550\end{array}$ & $\begin{array}{l}4.640 \\
6.640 \\
8.550\end{array}$ & $\begin{array}{l}2.450 \\
4.450 \\
6.450\end{array}$ \\
\hline LC42 & $\begin{array}{l}5.360 \\
7.360 \\
9.120\end{array}$ & $\begin{array}{l}5.120 \\
7.140 \\
8.720\end{array}$ & $\begin{array}{l}3.150 \\
5.150 \\
6.910\end{array}$ & $\begin{array}{l}2.820 \\
4.640 \\
6.640\end{array}$ & $\begin{array}{l}5.360 \\
7.360 \\
9.120\end{array}$ & $\begin{array}{l}1.450 \\
3.000 \\
4.910\end{array}$ & $\begin{array}{l}4.280 \\
6.370 \\
8.370\end{array}$ & $\begin{array}{l}2.450 \\
4.450 \\
6.450\end{array}$ & $\begin{array}{l}2.820 \\
4.640 \\
6.640\end{array}$ \\
\hline LC43 & $\begin{array}{l}4.280 \\
6.370 \\
8.370\end{array}$ & $\begin{array}{l}4.280 \\
6.370 \\
8.370\end{array}$ & $\begin{array}{l}2.450 \\
4.450 \\
6.450\end{array}$ & $\begin{array}{l}2.910 \\
4.640 \\
6.550\end{array}$ & $\begin{array}{l}4.640, \\
6.640, \\
8.550\end{array}$ & $\begin{array}{l}3.150 \\
5.150 \\
6.910\end{array}$ & $\begin{array}{l}2.820 \\
4.640 \\
6.640\end{array}$ & $\begin{array}{l}5.360 \\
7.360 \\
9.120\end{array}$ & $\begin{array}{l}1.180 \\
2.820 \\
4.820\end{array}$ \\
\hline LC44 & $\begin{array}{l}4.270 \\
6.270 \\
8.140\end{array}$ & $\begin{array}{l}2.910 \\
4.640 \\
6.550\end{array}$ & $\begin{array}{l}1.450 \\
3.000 \\
4.910\end{array}$ & $\begin{array}{l}4.280 \\
6.370 \\
8.370\end{array}$ & $\begin{array}{l}2.450 \\
4.450 \\
6.450\end{array}$ & $\begin{array}{l}2.450 \\
4.450 \\
6.450\end{array}$ & $\begin{array}{l}2.910 \\
4.640 \\
6.550\end{array}$ & $\begin{array}{l}4.640 \\
6.640 \\
8.550\end{array}$ & $\begin{array}{l}2.450 \\
4.450 \\
6.450\end{array}$ \\
\hline
\end{tabular}


Table 14. Cont.

\begin{tabular}{|c|c|c|c|c|c|c|c|c|c|}
\hline & AT1 & AT2 & AT3 & AT4 & AT5 & AT6 & AT7 & AT8 & AT9 \\
\hline LC45 & $\begin{array}{l}5.360, \\
7.360, \\
9.120\end{array}$ & $\begin{array}{l}5.120 \\
7.140 \\
8.720\end{array}$ & $\begin{array}{l}3.150 \\
5.150 \\
6.910\end{array}$ & $\begin{array}{l}2.820 \\
4.640 \\
6.640\end{array}$ & $\begin{array}{l}5.360 \\
7.360 \\
9.120\end{array}$ & $\begin{array}{l}1.450 \\
3.000 \\
4.910\end{array}$ & $\begin{array}{l}4.280 \\
6.370 \\
8.370\end{array}$ & $\begin{array}{l}2.450 \\
4.450 \\
6.450\end{array}$ & $\begin{array}{l}2.820 \\
4.640 \\
6.640\end{array}$ \\
\hline LC46 & $\begin{array}{l}4.280 \\
6.370 \\
8.370\end{array}$ & $\begin{array}{l}4.280 \\
6.370 \\
8.370\end{array}$ & $\begin{array}{l}2.450 \\
4.450 \\
6.450\end{array}$ & $\begin{array}{l}2.910 \\
4.640 \\
6.550\end{array}$ & $\begin{array}{l}4.640 \\
6.640 \\
8.550\end{array}$ & $\begin{array}{l}3.150 \\
5.150 \\
6.910\end{array}$ & $\begin{array}{l}2.820 \\
4.640 \\
6.640\end{array}$ & $\begin{array}{l}5.360 \\
7.360 \\
9.120\end{array}$ & $\begin{array}{l}1.180 \\
2.820 \\
4.820\end{array}$ \\
\hline LC47 & $\begin{array}{l}4.270 \\
6.270 \\
8.140\end{array}$ & $\begin{array}{l}2.910 \\
4.640 \\
6.550\end{array}$ & $\begin{array}{l}1.450 \\
3.000 \\
4.910\end{array}$ & $\begin{array}{l}4.280 \\
6.370 \\
8.370\end{array}$ & $\begin{array}{l}2.450 \\
4.450 \\
6.450\end{array}$ & $\begin{array}{l}2.450 \\
4.450 \\
6.450\end{array}$ & $\begin{array}{l}2.910, \\
4.640, \\
6.550\end{array}$ & $\begin{array}{l}4.640 \\
6.640 \\
8.550\end{array}$ & $\begin{array}{l}2.450 \\
4.450 \\
6.450\end{array}$ \\
\hline LC51 & $\begin{array}{l}5.360, \\
7.360 \\
9.120\end{array}$ & $\begin{array}{l}5.120 \\
7.140 \\
8.720\end{array}$ & $\begin{array}{l}3.150 \\
5.150 \\
6.910\end{array}$ & $\begin{array}{l}2.820 \\
4.640 \\
6.640\end{array}$ & $\begin{array}{l}5.360 \\
7.360 \\
9.120\end{array}$ & $\begin{array}{l}1.450 \\
3.000 \\
4.910\end{array}$ & $\begin{array}{l}4.280 \\
6.370 \\
8.370\end{array}$ & $\begin{array}{l}2.450 \\
4.450 \\
6.450\end{array}$ & $\begin{array}{l}2.820 \\
4.640 \\
6.640\end{array}$ \\
\hline LC52 & $\begin{array}{l}4.640, \\
6.640, \\
8.550\end{array}$ & $\begin{array}{l}4.280 \\
6.370 \\
8.370\end{array}$ & $\begin{array}{l}2.450 \\
4.450 \\
6.450\end{array}$ & $\begin{array}{l}2.910 \\
4.640 \\
6.550\end{array}$ & $\begin{array}{l}4.640, \\
6.640, \\
8.550\end{array}$ & $\begin{array}{l}3.150 \\
5.150 \\
6.910\end{array}$ & $\begin{array}{l}2.820 \\
4.640 \\
6.640\end{array}$ & $\begin{array}{l}5.360 \\
7.360 \\
9.120\end{array}$ & $\begin{array}{l}2.450 \\
4.450 \\
6.450\end{array}$ \\
\hline LC53 & $\begin{array}{l}3.120 \\
5.000 \\
7.140\end{array}$ & $\begin{array}{l}2.910 \\
4.640 \\
6.550\end{array}$ & $\begin{array}{l}1.450 \\
3.000 \\
4.910\end{array}$ & $\begin{array}{l}4.280 \\
6.370 \\
8.370\end{array}$ & $\begin{array}{l}2.450 \\
4.450 \\
6.450\end{array}$ & $\begin{array}{l}2.450 \\
4.450 \\
6.450\end{array}$ & $\begin{array}{l}2.910 \\
4.640 \\
6.550\end{array}$ & $\begin{array}{l}4.640 \\
6.640 \\
8.550\end{array}$ & $\begin{array}{l}2.450 \\
4.450 \\
6.450\end{array}$ \\
\hline LC54 & $\begin{array}{l}5.360, \\
7.360, \\
9.120\end{array}$ & $\begin{array}{l}5.120 \\
7.140 \\
8.720\end{array}$ & $\begin{array}{l}3.150 \\
5.150 \\
6.910\end{array}$ & $\begin{array}{l}2.820 \\
4.640 \\
6.640\end{array}$ & $\begin{array}{l}5.360, \\
7.360, \\
9.120\end{array}$ & $\begin{array}{l}2.820 \\
4.640 \\
6.640\end{array}$ & $\begin{array}{l}2.820 \\
4.640 \\
6.640\end{array}$ & $\begin{array}{l}5.360 \\
7.360 \\
9.120\end{array}$ & $\begin{array}{l}2.820 \\
4.640, \\
6.640\end{array}$ \\
\hline LC55 & $\begin{array}{l}4.640 \\
6.640 \\
8.550\end{array}$ & $\begin{array}{l}4.280 \\
6.370 \\
8.370\end{array}$ & $\begin{array}{l}2.450 \\
4.450 \\
6.450\end{array}$ & $\begin{array}{l}2.910 \\
4.640 \\
6.550\end{array}$ & $\begin{array}{l}4.640 \\
6.640 \\
8.550\end{array}$ & $\begin{array}{l}5.360 \\
7.360 \\
9.120\end{array}$ & $\begin{array}{l}3.730 \\
5.730 \\
7.550\end{array}$ & $\begin{array}{l}4.280 \\
6.370 \\
8.370\end{array}$ & $\begin{array}{l}2.450 \\
4.450 \\
6.450\end{array}$ \\
\hline
\end{tabular}

Table 15. The normalized fuzzy decision matrix.

\begin{tabular}{cccccccccc}
\hline & AT1 & AT2 & AT3 & AT4 & AT5 & AT6 & AT7 & AT8 & AT9 \\
\hline \multirow{2}{*}{ LC11 } & 0.460, & 0.320, & 0.390, & 0.460, & 0.320, & 0.390, & 0.210, & 0.180, & 0.210, \\
& 0.690, & 0.580, & 0.620, & 0.690, & 0.580, & 0.620, & 0.450, & 0.430, & 0.450, \\
& 0.910 & 0.850 & 0.870 & 0.910 & 0.850 & 0.870 & 0.730 & 0.740 & 0.730 \\
\hline \multirow{2}{*}{ LC12 } & 0.460, & 0.370, & 0.420, & 0.460, & 0.370, & 0.420, & 0.210, & 0.120, & 0.300, \\
& 0.680, & 0.630, & 0.690, & 0.680, & 0.630, & 0.690, & 0.460, & 0.350, & 0.530, \\
& 0.890 & 0.900 & 0.950 & 0.890 & 0.900 & 0.950 & 0.730 & 0.660 & 0.790 \\
\hline \multirow{2}{*}{ LC13 } & 0.560, & 0.410, & 0.370, & 0.560, & 0.410, & 0.370, & 0.230, & 0.220, & 0.260, \\
& 0.780, & 0.680, & 0.620, & 0.780, & 0.680, & 0.620, & 0.470, & 0.490, & 0.470, \\
& 0.950 & 0.910 & 0.890 & 0.950 & 0.910 & 0.890 & 0.780 & 0.800 & 0.720 \\
\hline \multirow{2}{*}{ LC21 } & 0.460, & 0.460, & 0.320, & 0.390, & 0.460, & 0.320, & 0.390, & 0.210, & 0.180, \\
& 0.690, & 0.690, & 0.580, & 0.620, & 0.690, & 0.580, & 0.620, & 0.450, & 0.430, \\
& 0.910 & 0.910 & 0.850 & 0.870 & 0.910 & 0.850 & 0.870 & 0.730 & 0.740 \\
\hline \multirow{2}{*}{ LC22 } & 0.460, & 0.460, & 0.370, & 0.420, & 0.460, & 0.370, & 0.420, & 0.210, & 0.120, \\
& 0.680, & 0.680, & 0.630, & 0.690, & 0.680, & 0.630, & 0.690, & 0.460, & 0.350, \\
& 0.890 & 0.890 & 0.900 & 0.950 & 0.890 & 0.900 & 0.950 & 0.730 & 0.660 \\
\hline \multirow{2}{*}{ LC31 } & 0.460, & 0.320, & 0.390, & 0.210, & 0.180, & 0.410, & 0.370, & 0.230, & 0.220, \\
& 0.690, & 0.580, & 0.620, & 0.450, & 0.430, & 0.680, & 0.620, & 0.470, & 0.490, \\
& 0.910 & 0.850 & 0.870 & 0.730 & 0.740 & 0.910 & 0.890 & 0.780 & 0.800 \\
\hline \multirow{2}{*}{ LC32 } & 0.460, & 0.370, & 0.420, & 0.210, & 0.460, & 0.320, & 0.390, & 0.210, & 0.180, \\
& 0.680, & 0.630, & 0.690, & 0.460, & 0.690, & 0.580, & 0.620, & 0.450, & 0.430, \\
& 0.890 & 0.900 & 0.950 & 0.730 & 0.910 & 0.850 & 0.870 & 0.730 & 0.740 \\
\hline
\end{tabular}


Table 15. Cont.

\begin{tabular}{|c|c|c|c|c|c|c|c|c|c|}
\hline & AT1 & AT2 & AT3 & AT4 & AT5 & AT6 & AT7 & AT8 & AT9 \\
\hline LC33 & $\begin{array}{l}0.560 \\
0.780 \\
0.950\end{array}$ & $\begin{array}{l}0.410 \\
0.680 \\
0.910\end{array}$ & $\begin{array}{l}0.370 \\
0.620 \\
0.890\end{array}$ & $\begin{array}{l}0.230 \\
0.470 \\
0.780\end{array}$ & $\begin{array}{l}0.460 \\
0.680 \\
0.890\end{array}$ & $\begin{array}{l}0.370 \\
0.630 \\
0.900\end{array}$ & $\begin{array}{l}0.420 \\
0.690 \\
0.950\end{array}$ & $\begin{array}{l}0.210 \\
0.460 \\
0.730\end{array}$ & $\begin{array}{l}0.120 \\
0.350 \\
0.660\end{array}$ \\
\hline LC34 & $\begin{array}{l}0.460 \\
0.690 \\
0.910\end{array}$ & $\begin{array}{l}0.320 \\
0.580 \\
0.850\end{array}$ & $\begin{array}{l}0.390 \\
0.620 \\
0.870\end{array}$ & $\begin{array}{l}0.210 \\
0.450 \\
0.730\end{array}$ & $\begin{array}{l}0.560 \\
0.780 \\
0.950\end{array}$ & $\begin{array}{l}0.410 \\
0.680 \\
0.910\end{array}$ & $\begin{array}{l}0.370 \\
0.620 \\
0.890\end{array}$ & $\begin{array}{l}0.230 \\
0.470 \\
0.780\end{array}$ & $\begin{array}{l}0.220 \\
0.490 \\
0.800\end{array}$ \\
\hline LC35 & $\begin{array}{l}0.460 \\
0.680 \\
0.890\end{array}$ & $\begin{array}{l}0.370 \\
0.630 \\
0.900\end{array}$ & $\begin{array}{l}0.420 \\
0.690 \\
0.950\end{array}$ & $\begin{array}{l}0.210 \\
0.460 \\
0.730\end{array}$ & $\begin{array}{l}0.460 \\
0.690 \\
0.910\end{array}$ & $\begin{array}{l}0.320 \\
0.580 \\
0.850\end{array}$ & $\begin{array}{l}0.390 \\
0.620 \\
0.870\end{array}$ & $\begin{array}{l}0.210 \\
0.450 \\
0.730\end{array}$ & $\begin{array}{l}0.180 \\
0.430 \\
0.740\end{array}$ \\
\hline LC36 & $\begin{array}{l}0.460 \\
0.690 \\
0.910\end{array}$ & $\begin{array}{l}0.320 \\
0.580 \\
0.850\end{array}$ & $\begin{array}{l}0.390 \\
0.620 \\
0.870\end{array}$ & $\begin{array}{l}0.210 \\
0.450 \\
0.730\end{array}$ & $\begin{array}{l}0.180 \\
0.430 \\
0.740\end{array}$ & $\begin{array}{l}0.460 \\
0.690 \\
0.910\end{array}$ & $\begin{array}{l}0.320 \\
0.580 \\
0.850\end{array}$ & $\begin{array}{l}0.390 \\
0.620 \\
0.870\end{array}$ & $\begin{array}{l}0.210 \\
0.450 \\
0.730\end{array}$ \\
\hline LC37 & $\begin{array}{l}0.460 \\
0.680 \\
0.890\end{array}$ & $\begin{array}{l}0.370 \\
0.630 \\
0.900\end{array}$ & $\begin{array}{l}0.420 \\
0.690 \\
0.950\end{array}$ & $\begin{array}{l}0.210 \\
0.460 \\
0.730\end{array}$ & $\begin{array}{l}0.120 \\
0.350 \\
0.660\end{array}$ & $\begin{array}{l}0.460 \\
0.680 \\
0.890\end{array}$ & $\begin{array}{l}0.370 \\
0.630 \\
0.900\end{array}$ & $\begin{array}{l}0.420 \\
0.690 \\
0.950\end{array}$ & $\begin{array}{l}0.210 \\
0.460 \\
0.730\end{array}$ \\
\hline LC38 & $\begin{array}{l}0.560 \\
0.780 \\
0.950\end{array}$ & $\begin{array}{l}0.410 \\
0.680 \\
0.910\end{array}$ & $\begin{array}{l}0.370 \\
0.620 \\
0.890\end{array}$ & $\begin{array}{l}0.230 \\
0.470 \\
0.780\end{array}$ & $\begin{array}{l}0.220 \\
0.490 \\
0.800\end{array}$ & $\begin{array}{l}0.560 \\
0.780 \\
0.950\end{array}$ & $\begin{array}{l}0.410 \\
0.680 \\
0.910\end{array}$ & $\begin{array}{l}0.370 \\
0.620 \\
0.890\end{array}$ & $\begin{array}{l}0.230 \\
0.470 \\
0.780\end{array}$ \\
\hline LC41 & $\begin{array}{l}0.460 \\
0.690 \\
0.910\end{array}$ & $\begin{array}{l}0.320 \\
0.580 \\
0.850\end{array}$ & $\begin{array}{l}0.390 \\
0.620 \\
0.870\end{array}$ & $\begin{array}{l}0.210 \\
0.450 \\
0.730\end{array}$ & $\begin{array}{l}0.180 \\
0.430 \\
0.740\end{array}$ & $\begin{array}{l}0.460 \\
0.690 \\
0.910\end{array}$ & $\begin{array}{l}0.320 \\
0.580 \\
0.850\end{array}$ & $\begin{array}{l}0.390 \\
0.620 \\
0.870\end{array}$ & $\begin{array}{l}0.210 \\
0.450 \\
0.730\end{array}$ \\
\hline LC42 & $\begin{array}{l}0.460 \\
0.680 \\
0.890\end{array}$ & $\begin{array}{l}0.370 \\
0.630 \\
0.900\end{array}$ & $\begin{array}{l}0.420 \\
0.690 \\
0.950\end{array}$ & $\begin{array}{l}0.210 \\
0.460 \\
0.730\end{array}$ & $\begin{array}{l}0.120 \\
0.350 \\
0.660\end{array}$ & $\begin{array}{l}0.460 \\
0.680 \\
0.890\end{array}$ & $\begin{array}{l}0.370 \\
0.630 \\
0.900\end{array}$ & $\begin{array}{l}0.420 \\
0.690 \\
0.950\end{array}$ & $\begin{array}{l}0.210 \\
0.460 \\
0.730\end{array}$ \\
\hline LC43 & $\begin{array}{l}0.230 \\
0.470 \\
0.780\end{array}$ & $\begin{array}{l}0.220 \\
0.490 \\
0.800\end{array}$ & $\begin{array}{l}0.460 \\
0.690 \\
0.910\end{array}$ & $\begin{array}{l}0.320 \\
0.580 \\
0.850\end{array}$ & $\begin{array}{l}0.390 \\
0.620 \\
0.870\end{array}$ & $\begin{array}{l}0.230 \\
0.470 \\
0.780\end{array}$ & $\begin{array}{l}0.220 \\
0.490 \\
0.800\end{array}$ & $\begin{array}{l}0.460 \\
0.690 \\
0.910\end{array}$ & $\begin{array}{l}0.320 \\
0.580 \\
0.850\end{array}$ \\
\hline LC44 & $\begin{array}{l}0.560 \\
0.780 \\
0.950\end{array}$ & $\begin{array}{l}0.410 \\
0.680 \\
0.910\end{array}$ & $\begin{array}{l}0.370 \\
0.620 \\
0.890\end{array}$ & $\begin{array}{l}0.230 \\
0.470 \\
0.780\end{array}$ & $\begin{array}{l}0.220 \\
0.490 \\
0.800\end{array}$ & $\begin{array}{l}0.560 \\
0.780 \\
0.950\end{array}$ & $\begin{array}{l}0.410 \\
0.680 \\
0.910\end{array}$ & $\begin{array}{l}0.370 \\
0.620 \\
0.890\end{array}$ & $\begin{array}{l}0.230 \\
0.470 \\
0.780\end{array}$ \\
\hline LC45 & $\begin{array}{l}0.460 \\
0.690 \\
0.910\end{array}$ & $\begin{array}{l}0.320 \\
0.580 \\
0.850\end{array}$ & $\begin{array}{l}0.390 \\
0.620 \\
0.870\end{array}$ & $\begin{array}{l}0.210 \\
0.450 \\
0.730\end{array}$ & $\begin{array}{l}0.180 \\
0.430 \\
0.740\end{array}$ & $\begin{array}{l}0.460 \\
0.690 \\
0.910\end{array}$ & $\begin{array}{l}0.320 \\
0.580 \\
0.850\end{array}$ & $\begin{array}{l}0.390 \\
0.620 \\
0.870\end{array}$ & $\begin{array}{l}0.210 \\
0.450 \\
0.730\end{array}$ \\
\hline LC46 & $\begin{array}{l}0.460 \\
0.690 \\
0.910\end{array}$ & $\begin{array}{l}0.320 \\
0.580 \\
0.850\end{array}$ & $\begin{array}{l}0.390 \\
0.620 \\
0.870\end{array}$ & $\begin{array}{l}0.210 \\
0.450 \\
0.730\end{array}$ & $\begin{array}{l}0.460 \\
0.690 \\
0.910\end{array}$ & $\begin{array}{l}0.320 \\
0.580 \\
0.850\end{array}$ & $\begin{array}{l}0.390 \\
0.620 \\
0.870\end{array}$ & $\begin{array}{l}0.210 \\
0.450 \\
0.730\end{array}$ & $\begin{array}{l}0.180 \\
0.430 \\
0.740\end{array}$ \\
\hline LC47 & $\begin{array}{l}0.460 \\
0.680 \\
0.890\end{array}$ & $\begin{array}{l}0.370 \\
0.630 \\
0.900\end{array}$ & $\begin{array}{l}0.420 \\
0.690 \\
0.950\end{array}$ & $\begin{array}{l}0.210 \\
0.460 \\
0.730\end{array}$ & $\begin{array}{l}0.460 \\
0.680 \\
0.890\end{array}$ & $\begin{array}{l}0.370 \\
0.630 \\
0.900\end{array}$ & $\begin{array}{l}0.420 \\
0.690 \\
0.950\end{array}$ & $\begin{array}{l}0.210 \\
0.460 \\
0.730\end{array}$ & $\begin{array}{l}0.120 \\
0.350 \\
0.660\end{array}$ \\
\hline LC51 & $\begin{array}{l}0.560 \\
0.780 \\
0.950\end{array}$ & $\begin{array}{l}0.410 \\
0.680 \\
0.910\end{array}$ & $\begin{array}{l}0.370 \\
0.620 \\
0.890\end{array}$ & $\begin{array}{l}0.230 \\
0.470 \\
0.780\end{array}$ & $\begin{array}{l}0.560 \\
0.780 \\
0.950\end{array}$ & $\begin{array}{l}0.410 \\
0.680 \\
0.910\end{array}$ & $\begin{array}{l}0.370 \\
0.620 \\
0.890\end{array}$ & $\begin{array}{l}0.230 \\
0.470 \\
0.780\end{array}$ & $\begin{array}{l}0.220 \\
0.490 \\
0.800\end{array}$ \\
\hline LC52 & $\begin{array}{l}0.460 \\
0.690 \\
0.910\end{array}$ & $\begin{array}{l}0.320 \\
0.580 \\
0.850\end{array}$ & $\begin{array}{l}0.390 \\
0.620 \\
0.870\end{array}$ & $\begin{array}{l}0.210 \\
0.450 \\
0.730\end{array}$ & $\begin{array}{l}0.460 \\
0.690 \\
0.910\end{array}$ & $\begin{array}{l}0.320 \\
0.580 \\
0.850\end{array}$ & $\begin{array}{l}0.390 \\
0.620 \\
0.870\end{array}$ & $\begin{array}{l}0.210 \\
0.450 \\
0.730\end{array}$ & $\begin{array}{l}0.180 \\
0.430 \\
0.740\end{array}$ \\
\hline LC53 & $\begin{array}{l}0.460 \\
0.680 \\
0.890\end{array}$ & $\begin{array}{l}0.370 \\
0.630 \\
0.900\end{array}$ & $\begin{array}{l}0.420 \\
0.690 \\
0.950\end{array}$ & $\begin{array}{l}0.210 \\
0.460 \\
0.730\end{array}$ & $\begin{array}{l}0.460 \\
0.680 \\
0.890\end{array}$ & $\begin{array}{l}0.370 \\
0.630 \\
0.900\end{array}$ & $\begin{array}{l}0.420 \\
0.690 \\
0.950\end{array}$ & $\begin{array}{l}0.210 \\
0.460 \\
0.730\end{array}$ & $\begin{array}{l}0.120 \\
0.350 \\
0.660\end{array}$ \\
\hline
\end{tabular}


Table 15. Cont

\begin{tabular}{cccccccccc}
\hline & AT1 & AT2 & AT3 & AT4 & AT5 & AT6 & AT7 & AT8 & AT9 \\
\hline \multirow{4}{*}{ LC54 } & 0.230, & 0.220, & 0.460, & 0.320, & 0.230, & 0.220, & 0.460, & 0.320, & 0.390, \\
& 0.470, & 0.490, & 0.690, & 0.580, & 0.470, & 0.490, & 0.690, & 0.580, & 0.620, \\
& 0.780 & 0.800 & 0.910 & 0.850 & 0.780 & 0.800 & 0.910 & 0.850 & 0.870 \\
\hline \multirow{3}{*}{ LC55 } & 0.560, & 0.410, & 0.370, & 0.230, & 0.560, & 0.410, & 0.370, & 0.230, & 0.220, \\
& 0.780, & 0.680, & 0.620, & 0.470, & 0.780, & 0.680, & 0.620, & 0.470, & 0.490, \\
& 0.950 & 0.910 & 0.890 & 0.780 & 0.950 & 0.910 & 0.890 & 0.780 & 0.800 \\
\hline
\end{tabular}

Table 16. The weighted normalized fuzzy decision matrix.

\begin{tabular}{|c|c|c|c|c|c|c|c|c|c|}
\hline & AT1 & AT2 & AT3 & AT4 & AT5 & AT6 & AT7 & AT8 & AT9 \\
\hline LC11 & $\begin{array}{l}0.054, \\
0.120 \\
0.260\end{array}$ & $\begin{array}{l}0.125 \\
0.155 \\
0.344\end{array}$ & $\begin{array}{l}0.041 \\
0.095 \\
0.242\end{array}$ & $\begin{array}{l}0.059 \\
0.121 \\
0.296\end{array}$ & $\begin{array}{l}0.041 \\
0.100 \\
0.260\end{array}$ & $\begin{array}{l}0.045 \\
0.098 \\
0.239\end{array}$ & $\begin{array}{l}0.041 \\
0.095 \\
0.242\end{array}$ & $\begin{array}{l}0.041 \\
0.100 \\
0.260\end{array}$ & $\begin{array}{l}0.045, \\
0.098, \\
0.239\end{array}$ \\
\hline LC12 & $\begin{array}{l}0.043, \\
0.096, \\
0.196\end{array}$ & $\begin{array}{l}0.041 \\
0.095 \\
0.198\end{array}$ & $\begin{array}{l}0.061, \\
0.121 \\
0.233\end{array}$ & $\begin{array}{l}0.125, \\
0.155, \\
0.344\end{array}$ & $\begin{array}{l}0.041, \\
0.095, \\
0.242\end{array}$ & $\begin{array}{l}0.059 \\
0.121 \\
0.296\end{array}$ & $\begin{array}{l}0.041 \\
0.100 \\
0.260\end{array}$ & $\begin{array}{l}0.045, \\
0.098 \\
0.239\end{array}$ & $\begin{array}{l}0.041, \\
0.095, \\
0.242\end{array}$ \\
\hline LC13 & $\begin{array}{l}0.041, \\
0.095 \\
0.242\end{array}$ & $\begin{array}{l}0.102, \\
0.137 \\
0.299\end{array}$ & $\begin{array}{l}0.114 \\
0.144 \\
0.306\end{array}$ & $\begin{array}{l}0.041 \\
0.095 \\
0.198\end{array}$ & $\begin{array}{l}0.061 \\
0.121 \\
0.233\end{array}$ & $\begin{array}{l}0.114 \\
0.144, \\
0.306\end{array}$ & $\begin{array}{l}0.125, \\
0.155, \\
0.344\end{array}$ & $\begin{array}{l}0.041 \\
0.095, \\
0.242\end{array}$ & $\begin{array}{l}0.059, \\
0.121 \\
0.296\end{array}$ \\
\hline LC21 & $\begin{array}{l}0.061, \\
0.121, \\
0.233\end{array}$ & $\begin{array}{l}0.027 \\
0.080 \\
0.197\end{array}$ & $\begin{array}{l}0.051 \\
0.104, \\
0.168\end{array}$ & $\begin{array}{l}0.102, \\
0.137 \\
0.299\end{array}$ & $\begin{array}{l}0.114, \\
0.144, \\
0.306\end{array}$ & $\begin{array}{l}0.044, \\
0.088, \\
0.182\end{array}$ & $\begin{array}{l}0.041, \\
0.095, \\
0.198\end{array}$ & $\begin{array}{l}0.061, \\
0.121 \\
0.233\end{array}$ & $\begin{array}{l}0.034, \\
0.091 \\
0.200\end{array}$ \\
\hline LC22 & $\begin{array}{l}0.125, \\
0.155, \\
0.344\end{array}$ & $\begin{array}{l}0.041 \\
0.095, \\
0.242\end{array}$ & $\begin{array}{l}0.059 \\
0.121 \\
0.296\end{array}$ & $\begin{array}{l}0.041 \\
0.100 \\
0.260\end{array}$ & $\begin{array}{l}0.045, \\
0.098, \\
0.239\end{array}$ & $\begin{array}{l}0.041, \\
0.095, \\
0.242\end{array}$ & $\begin{array}{l}0.125, \\
0.155, \\
0.344\end{array}$ & $\begin{array}{l}0.041, \\
0.095, \\
0.242\end{array}$ & $\begin{array}{l}0.059 \\
0.121 \\
0.296\end{array}$ \\
\hline LC31 & $\begin{array}{l}0.041 \\
0.095 \\
0.198\end{array}$ & $\begin{array}{l}0.061 \\
0.121 \\
0.233\end{array}$ & $\begin{array}{l}0.114 \\
0.144 \\
0.306\end{array}$ & $\begin{array}{l}0.125, \\
0.155 \\
0.344\end{array}$ & $\begin{array}{l}0.125, \\
0.155 \\
0.344\end{array}$ & $\begin{array}{l}0.041 \\
0.095 \\
0.242\end{array}$ & $\begin{array}{l}0.059 \\
0.121 \\
0.296\end{array}$ & $\begin{array}{c}0.041 \\
0.100 \\
0.260\end{array}$ & $\begin{array}{c}0.045 \\
0.098 \\
0.239\end{array}$ \\
\hline LC32 & $\begin{array}{l}0.102, \\
0.137 \\
0.299\end{array}$ & $\begin{array}{l}0.114 \\
0.144 \\
0.306\end{array}$ & $\begin{array}{l}0.044, \\
0.088, \\
0.182\end{array}$ & $\begin{array}{l}0.041 \\
0.095 \\
0.198\end{array}$ & $\begin{array}{l}0.041 \\
0.095 \\
0.198\end{array}$ & $\begin{array}{l}0.061, \\
0.121, \\
0.233\end{array}$ & $\begin{array}{l}0.114, \\
0.144, \\
0.306\end{array}$ & $\begin{array}{l}0.125, \\
0.155, \\
0.344\end{array}$ & $\begin{array}{l}0.041, \\
0.095 \\
0.242\end{array}$ \\
\hline LC33 & $\begin{array}{l}0.125, \\
0.155, \\
0.344\end{array}$ & $\begin{array}{l}0.041 \\
0.095 \\
0.242\end{array}$ & $\begin{array}{l}0.059 \\
0.121 \\
0.296\end{array}$ & $\begin{array}{l}0.041 \\
0.100 \\
0.260\end{array}$ & $\begin{array}{l}0.045, \\
0.098, \\
0.239\end{array}$ & $\begin{array}{l}0.041, \\
0.095, \\
0.242\end{array}$ & $\begin{array}{l}0.044, \\
0.088, \\
0.182\end{array}$ & $\begin{array}{l}0.041, \\
0.095, \\
0.198\end{array}$ & $\begin{array}{l}0.061, \\
0.121 \\
0.233\end{array}$ \\
\hline LC34 & $\begin{array}{l}0.041 \\
0.095 \\
0.198\end{array}$ & $\begin{array}{l}0.061, \\
0.121 \\
0.233\end{array}$ & $\begin{array}{l}0.114, \\
0.144, \\
0.306\end{array}$ & $\begin{array}{l}0.125, \\
0.155, \\
0.344\end{array}$ & $\begin{array}{l}0.041, \\
0.095, \\
0.242\end{array}$ & $\begin{array}{l}0.059 \\
0.121 \\
0.296\end{array}$ & $\begin{array}{l}0.114, \\
0.144, \\
0.306\end{array}$ & $\begin{array}{l}0.125, \\
0.155, \\
0.344\end{array}$ & $\begin{array}{l}0.041, \\
0.095 \\
0.242\end{array}$ \\
\hline LC35 & $\begin{array}{l}0.102, \\
0.137 \\
0.299\end{array}$ & $\begin{array}{l}0.114 \\
0.144 \\
0.306\end{array}$ & $\begin{array}{l}0.044, \\
0.088, \\
0.182\end{array}$ & $\begin{array}{l}0.125, \\
0.155, \\
0.344\end{array}$ & $\begin{array}{l}0.041 \\
0.095 \\
0.242\end{array}$ & $\begin{array}{l}0.059 \\
0.121 \\
0.296\end{array}$ & $\begin{array}{l}0.041 \\
0.100 \\
0.260\end{array}$ & $\begin{array}{l}0.045 \\
0.098 \\
0.239\end{array}$ & $\begin{array}{l}0.041 \\
0.095 \\
0.242\end{array}$ \\
\hline LC36 & $\begin{array}{l}0.027 \\
0.080 \\
0.197\end{array}$ & $\begin{array}{l}0.051 \\
0.104 \\
0.168\end{array}$ & $\begin{array}{l}0.114 \\
0.144 \\
0.306\end{array}$ & $\begin{array}{l}0.041 \\
0.095 \\
0.198\end{array}$ & $\begin{array}{l}0.061 \\
0.121 \\
0.233\end{array}$ & $\begin{array}{l}0.114, \\
0.144, \\
0.306\end{array}$ & $\begin{array}{l}0.125, \\
0.155, \\
0.344\end{array}$ & $\begin{array}{l}0.041 \\
0.095 \\
0.242\end{array}$ & $\begin{array}{l}0.059, \\
0.121 \\
0.296\end{array}$ \\
\hline LC37 & $\begin{array}{l}0.054, \\
0.120 \\
0.260\end{array}$ & $\begin{array}{l}0.027 \\
0.080 \\
0.197\end{array}$ & $\begin{array}{l}0.051, \\
0.104, \\
0.168\end{array}$ & $\begin{array}{l}0.102, \\
0.137 \\
0.299\end{array}$ & $\begin{array}{l}0.114, \\
0.144, \\
0.306\end{array}$ & $\begin{array}{l}0.044, \\
0.088, \\
0.182\end{array}$ & $\begin{array}{l}0.041 \\
0.095 \\
0.198\end{array}$ & $\begin{array}{l}0.061, \\
0.121 \\
0.233\end{array}$ & $\begin{array}{l}0.034, \\
0.091 \\
0.200\end{array}$ \\
\hline LC38 & $\begin{array}{l}0.125 \\
0.155 \\
0.344\end{array}$ & $\begin{array}{l}0.041 \\
0.095 \\
0.242\end{array}$ & $\begin{array}{l}0.059 \\
0.121 \\
0.296\end{array}$ & $\begin{array}{l}0.041 \\
0.100 \\
0.260\end{array}$ & $\begin{array}{l}0.045 \\
0.098 \\
0.239\end{array}$ & $\begin{array}{l}0.041 \\
0.095 \\
0.242\end{array}$ & $\begin{array}{l}0.125 \\
0.155 \\
0.344\end{array}$ & $\begin{array}{l}0.041 \\
0.095 \\
0.242\end{array}$ & $\begin{array}{l}0.059 \\
0.121 \\
0.296\end{array}$ \\
\hline
\end{tabular}


Table 16. Cont.

\begin{tabular}{|c|c|c|c|c|c|c|c|c|c|}
\hline & AT1 & AT2 & AT3 & AT4 & AT5 & AT6 & AT7 & AT8 & AT9 \\
\hline LC41 & $\begin{array}{l}0.041 \\
0.095 \\
0.198\end{array}$ & $\begin{array}{l}0.061 \\
0.121 \\
0.233\end{array}$ & $\begin{array}{l}0.114 \\
0.144 \\
0.306\end{array}$ & $\begin{array}{l}0.125 \\
0.155 \\
0.344\end{array}$ & $\begin{array}{l}0.125 \\
0.155 \\
0.344\end{array}$ & $\begin{array}{l}0.041 \\
0.095 \\
0.242\end{array}$ & $\begin{array}{l}0.059 \\
0.121 \\
0.296\end{array}$ & $\begin{array}{l}0.041 \\
0.100 \\
0.260\end{array}$ & $\begin{array}{l}0.045 \\
0.098 \\
0.239\end{array}$ \\
\hline LC42 & $\begin{array}{l}0.102 \\
0.137 \\
0.299\end{array}$ & $\begin{array}{l}0.114 \\
0.144 \\
0.306\end{array}$ & $\begin{array}{l}0.044 \\
0.088 \\
0.182\end{array}$ & $\begin{array}{l}0.041 \\
0.095 \\
0.198\end{array}$ & $\begin{array}{c}0.041 \\
0.095 \\
0.198\end{array}$ & $\begin{array}{l}0.061 \\
0.121 \\
0.233\end{array}$ & $\begin{array}{l}0.114 \\
0.144 \\
0.306\end{array}$ & $\begin{array}{l}0.125 \\
0.155 \\
0.344\end{array}$ & $\begin{array}{l}0.041 \\
0.095 \\
0.242\end{array}$ \\
\hline LC43 & $\begin{array}{l}0.027 \\
0.080 \\
0.197\end{array}$ & $\begin{array}{l}0.051 \\
0.104 \\
0.168\end{array}$ & $\begin{array}{l}0.114 \\
0.144 \\
0.306\end{array}$ & $\begin{array}{l}0.125 \\
0.155 \\
0.344\end{array}$ & $\begin{array}{l}0.102, \\
0.137 \\
0.299\end{array}$ & $\begin{array}{l}0.114 \\
0.144 \\
0.306\end{array}$ & $\begin{array}{l}0.044, \\
0.088 \\
0.182\end{array}$ & $\begin{array}{l}0.041 \\
0.095 \\
0.198\end{array}$ & $\begin{array}{l}0.061 \\
0.121 \\
0.233\end{array}$ \\
\hline LC44 & $\begin{array}{l}0.043 \\
0.096 \\
0.196\end{array}$ & $\begin{array}{l}0.017 \\
0.059 \\
0.152\end{array}$ & $\begin{array}{l}0.036 \\
0.072 \\
0.162\end{array}$ & $\begin{array}{l}0.044 \\
0.088 \\
0.182\end{array}$ & $\begin{array}{l}0.027 \\
0.080 \\
0.197\end{array}$ & $\begin{array}{l}0.051 \\
0.104 \\
0.168\end{array}$ & $\begin{array}{l}0.114 \\
0.144, \\
0.306\end{array}$ & $\begin{array}{l}0.125, \\
0.155, \\
0.344\end{array}$ & $\begin{array}{l}0.041 \\
0.095 \\
0.242\end{array}$ \\
\hline LC45 & $\begin{array}{l}0.125 \\
0.155 \\
0.344\end{array}$ & $\begin{array}{l}0.041 \\
0.095 \\
0.242\end{array}$ & $\begin{array}{l}0.059 \\
0.121 \\
0.296\end{array}$ & $\begin{array}{l}0.041 \\
0.100 \\
0.260\end{array}$ & $\begin{array}{l}0.125 \\
0.155 \\
0.344\end{array}$ & $\begin{array}{l}0.041 \\
0.095 \\
0.242\end{array}$ & $\begin{array}{l}0.059 \\
0.121 \\
0.296\end{array}$ & $\begin{array}{l}0.041 \\
0.100 \\
0.260\end{array}$ & $\begin{array}{l}0.045 \\
0.098 \\
0.239\end{array}$ \\
\hline LC46 & $\begin{array}{l}0.041 \\
0.095 \\
0.198\end{array}$ & $\begin{array}{l}0.061 \\
0.121 \\
0.233\end{array}$ & $\begin{array}{l}0.114 \\
0.144 \\
0.306\end{array}$ & $\begin{array}{l}0.125 \\
0.155 \\
0.344\end{array}$ & $\begin{array}{c}0.041 \\
0.095 \\
0.198\end{array}$ & $\begin{array}{l}0.061 \\
0.121 \\
0.233\end{array}$ & $\begin{array}{c}0.114 \\
0.144 \\
0.306\end{array}$ & $\begin{array}{l}0.125 \\
0.155 \\
0.344\end{array}$ & $\begin{array}{l}0.041 \\
0.095 \\
0.242\end{array}$ \\
\hline LC47 & $\begin{array}{l}0.102 \\
0.137 \\
0.299\end{array}$ & $\begin{array}{l}0.114 \\
0.144 \\
0.306\end{array}$ & $\begin{array}{l}0.044 \\
0.088 \\
0.182\end{array}$ & $\begin{array}{l}0.041 \\
0.095 \\
0.198\end{array}$ & $\begin{array}{l}0.102 \\
0.137 \\
0.299\end{array}$ & $\begin{array}{l}0.114 \\
0.144 \\
0.306\end{array}$ & $\begin{array}{c}0.044 \\
0.088 \\
0.182\end{array}$ & $\begin{array}{l}0.041 \\
0.095 \\
0.198\end{array}$ & $\begin{array}{l}0.061 \\
0.121 \\
0.233\end{array}$ \\
\hline LC51 & $\begin{array}{l}0.125 \\
0.155 \\
0.344\end{array}$ & $\begin{array}{l}0.041 \\
0.095 \\
0.242\end{array}$ & $\begin{array}{l}0.059 \\
0.121 \\
0.296\end{array}$ & $\begin{array}{l}0.041 \\
0.100 \\
0.260\end{array}$ & $\begin{array}{l}0.125 \\
0.155 \\
0.344\end{array}$ & $\begin{array}{l}0.041 \\
0.095 \\
0.242\end{array}$ & $\begin{array}{l}0.059 \\
0.121 \\
0.296\end{array}$ & $\begin{array}{l}0.041 \\
0.100 \\
0.260\end{array}$ & $\begin{array}{l}0.045 \\
0.098 \\
0.239\end{array}$ \\
\hline LC52 & $\begin{array}{l}0.041 \\
0.095 \\
0.198\end{array}$ & $\begin{array}{l}0.061 \\
0.121 \\
0.233\end{array}$ & $\begin{array}{l}0.114 \\
0.144 \\
0.306\end{array}$ & $\begin{array}{l}0.125 \\
0.155 \\
0.344\end{array}$ & $\begin{array}{l}0.041 \\
0.095 \\
0.198\end{array}$ & $\begin{array}{l}0.061 \\
0.121 \\
0.233\end{array}$ & $\begin{array}{l}0.114 \\
0.144 \\
0.306\end{array}$ & $\begin{array}{l}0.125 \\
0.155 \\
0.344\end{array}$ & $\begin{array}{l}0.041 \\
0.095 \\
0.242\end{array}$ \\
\hline LC53 & $\begin{array}{l}0.102 \\
0.137 \\
0.299\end{array}$ & $\begin{array}{l}0.114 \\
0.144 \\
0.306\end{array}$ & $\begin{array}{l}0.044 \\
0.088 \\
0.182\end{array}$ & $\begin{array}{l}0.041 \\
0.095 \\
0.198\end{array}$ & $\begin{array}{l}0.102 \\
0.137 \\
0.299\end{array}$ & $\begin{array}{l}0.114 \\
0.144 \\
0.306\end{array}$ & $\begin{array}{l}0.044, \\
0.088 \\
0.182\end{array}$ & $\begin{array}{l}0.041 \\
0.095 \\
0.198\end{array}$ & $\begin{array}{l}0.061 \\
0.121 \\
0.233\end{array}$ \\
\hline LC54 & $\begin{array}{l}0.027 \\
0.080 \\
0.197\end{array}$ & $\begin{array}{l}0.051 \\
0.104 \\
0.168\end{array}$ & $\begin{array}{l}0.114 \\
0.144 \\
0.306\end{array}$ & $\begin{array}{l}0.125 \\
0.155 \\
0.344\end{array}$ & $\begin{array}{l}0.027 \\
0.080 \\
0.197\end{array}$ & $\begin{array}{l}0.051 \\
0.104 \\
0.168\end{array}$ & $\begin{array}{l}0.114 \\
0.144 \\
0.306\end{array}$ & $\begin{array}{l}0.125 \\
0.155 \\
0.344\end{array}$ & $\begin{array}{l}0.041 \\
0.095 \\
0.242\end{array}$ \\
\hline LC55 & $\begin{array}{l}0.043 \\
0.096 \\
0.196\end{array}$ & $\begin{array}{l}0.017 \\
0.059 \\
0.152\end{array}$ & $\begin{array}{l}0.036 \\
0.072 \\
0.162\end{array}$ & $\begin{array}{l}0.044 \\
0.088 \\
0.182\end{array}$ & $\begin{array}{l}0.041 \\
0.095 \\
0.198\end{array}$ & $\begin{array}{l}0.061 \\
0.121 \\
0.233\end{array}$ & $\begin{array}{l}0.034 \\
0.091 \\
0.200\end{array}$ & $\begin{array}{l}0.032 \\
0.089 \\
0.200\end{array}$ & $\begin{array}{l}0.063 \\
0.120 \\
0.233\end{array}$ \\
\hline
\end{tabular}

Table 17. Closeness coefficients to the aspired level among the different alternatives.

\begin{tabular}{ccccc}
\hline Alternatives & $\mathbf{d}+\mathbf{i}$ & $\mathbf{d}-\mathbf{i}$ & Gap Degree of CC+i & Satisfaction Degree of CC-i \\
\hline AT1 & 1.249124 & 1.333548 & 0.5164578 & 0.4832657 \\
AT2 & 0.699528 & 0.840586 & 0.5476598 & 0.4532564 \\
AT3 & 0.787654 & 1.484745 & 0.6535644 & 0.3452657 \\
AT4 & 2.168547 & 1.484856 & 0.4125471 & 0.5923547 \\
AT5 & 2.005654 & 1.536954 & 0.4332654 & 0.5652547 \\
AT6 & 0.445476 & 0.392356 & 0.4645687 & 0.5345854 \\
AT7 & 0.788574 & 1.484657 & 0.4125475 & 0.4522564 \\
AT8 & 2.160256 & 1.536235 & 0.4332654 & 0.3452544 \\
AT9 & 2.035657 & 0.397596 & 0.4645288 & 0.5912556 \\
\hline
\end{tabular}




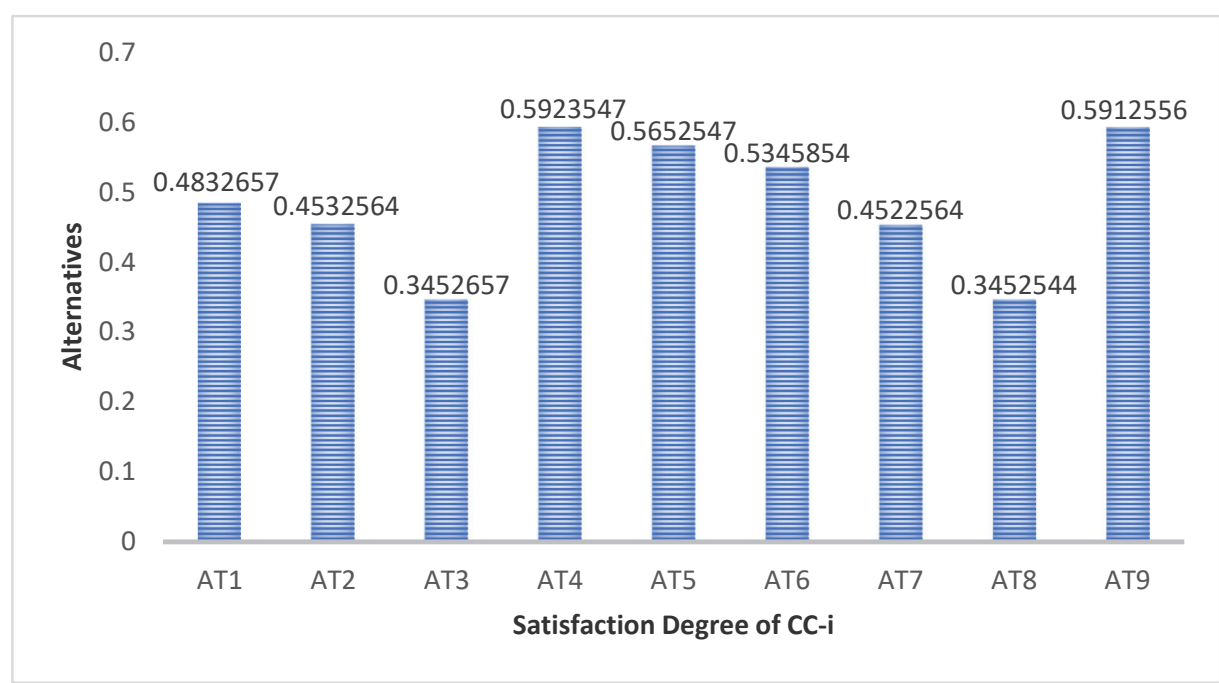

Figure 5. Graphical representation of closeness coefficients to the aspired level among the different alternatives.

Consequently, RAFAALS (denoted by AT4) was found to be best among nine comparative alternatives because it offers the best functionality with an efficiency score of 0.5923547 among the different Ambient Assisted Living system's symmetrical models. Alternative AT4 was followed by AT9, AT5, AT6, AT1, AT2, AT7, AT3, and AT8 with performance scores of $0.5912556,0.5652547,0.5345854,0.4832657,0.4532564,0.4522564,0.3452657$ and 0.3452544 , respectively.

\section{Conclusions}

AAL symmetrical models have progressed as an outcome of the emergence of global population ageing and the change of direction of technological advances. It lies at the intersection of technological innovation and age advancement. This multidisciplinary area of scientific research sees technology as a way of enhancing the lives of older people and promoting their involvement as involved members of society. This paper explores the principles of the Ambient Assisted Living area. We tested predefined categories to illustrate the key areas that need to change due to the advancement of AAL systems. We suggest that there is a lack of standardization in several of the available frameworks. This is the key deterrent in achieving the desired efficacy of the models. Maintenance and interoperability occur in all of the models. In some systems, there is a need for facility, interoperability, and independence. For a specific design model, the practitioners need to focus on a solid standard and develop a robust infrastructure. One of the crucial aspects of AAL systems is coping with data flow, which remains a largely unexplored domain and needs more dedicated research.

Author Contributions: W.A. performed formal analysis and investigation. M.T.J.A. conceived and designed the experiments, performed the computation work authored and reviewed drafts of the paper. A.A. (Abdullah Alharbi) contributed reagents/materials/analysis tools. H.A. performed the visualization, review and editing work. A.H.S. analyzed and reviewed the drafts. A.K.P. performed the review and editing work. A.A. (Alka Agrawal) analyzed the data, contributed reagents/materials/analysis tools, authored or reviewed drafts of the paper. R.A.K. conceived and designed the experiments, performed the experiments, contributed reagents/materials/analysis tools. All authors have read and agreed to the published version of the manuscript.

Funding: The project has been funded by Taif University, Kingdom of Saudi Arabia.

Institutional Review Board Statement: Not applicable.

Informed Consent Statement: Not applicable. 
Data Availability Statement: Not applicable.

Acknowledgments: This research was supported by Taif University Researchers Supporting Project number (TURSP-2020/254), Taif University, Taif, Saudi Arabia.

Conflicts of Interest: The authors declare no conflict of interest.

\section{References}

1. Chuah, S.H.-W.; Rauschnabel, P.A.; Krey, N.; Nguyen, B.; Ramayah, T.; Lade, S. Wearable technologies: The role of usefulness and visibility in smartwatch adoption. Comput. Hum. Behav. 2016, 65, 276-284. [CrossRef]

2. National Research Council. Preparing for an Aging World: The Case for Cross-National Research; National Academies Press: Washington, DC, USA, 2001.

3. Sun, H.; De Florio, V.; Gui, N.; Blondia, C. Promises and challenges of ambient assisted living systems. In Proceedings of the 2009 Sixth International Conference on Information Technology: New Generations, Las Vegas, NV, USA, 27-29 April 2009; pp. 1201-1207.

4. Rashidi, P.; Mihailidis, A. A survey on ambient-assisted living tools for older adults. IEEE J. Biomed. Health Inform. 2012, 17, 579-590. [CrossRef]

5. Freitas, P.; Menezes, P.; Dias, J. Ambient Assisted Living_From Technology to Intervention. In Ambient Assisted Living; CRC Press: Boca Raton, FL, USA, 2015; pp. 384-421.

6. Stopczynski, A.; Stahlhut, C.; Larsen, J.E.; Petersen, M.K.; Hansen, L.K. The Smartphone Brain Scanner: A Portable Real-Time Neuroimaging System. PLoS ONE 2014, 9, e86733. [CrossRef] [PubMed]

7. Memon, M.; Wagner, S.R.; Pedersen, C.F.; Beevi, F.H.A.; Hansen, F.O. Ambient Assisted Living Healthcare Frameworks, Platforms, Standards, and Quality Attributes. Sensors 2014, 14, 4312-4341. [CrossRef]

8. Allen, N.B.; Nelson, B.W.; Brent, D.; Auerbach, R.P. Short-term prediction of suicidal thoughts and behaviors in adolescents: Can recent developments in technology and computational science provide a breakthrough? J. Affect. Disord. 2019, 250, 163-169. [CrossRef] [PubMed]

9. Abtoy, A.; Touhafi, A.; Tahiri, A. Ambient Assisted living system's models and architectures: A survey of the state of the art. J. King Saud Univ. Comput. Inf. Sci. 2020, 32, 1-10.

10. Ghodsypour, S.H.; O’Brien, C. A decision support system for supplier selection using an integrated analytic hierarchy process and linear programming. Int. J. Prod. Econ. 1998, 56, 199-212. [CrossRef]

11. Carney, D.J.; Wallnau, K.C. A basis for evaluation of commercial software. Inf. Softw. Technol. 1998, 40, 851-860. [CrossRef]

12. Hwang, C.L.; Yoon, K. Multiple Attribute Decision Making; Springer: Berlin/Heidelberg, Germany, 1981.

13. Dursun, M.; Karsak, E.E. A fuzzy MCDM approach for personnel selection. Expert Syst. Appl. 2010, 37, 4324-4330. [CrossRef]

14. Lin, Y.-C.; Wang, Y.-C.; Chen, T.-C.T.; Lin, H.-F. Evaluating the Suitability of a Smart Technology Application for Fall Detection Using a Fuzzy Collaborative Intelligence Approach. Mathematics 2019, 7, 1097. [CrossRef]

15. Samanlioglu, F.; Taskaya, Y.E.; Gulen, U.C.; Cokcan, O. A fuzzy AHP-TOPSIS-based group decision-making approach to IT personnel selection. Int. J. Fuzzy Syst. 2018, 20, 1576-1591. [CrossRef]

16. Anand, M.B.; Vinodh, S. Application of fuzzy AHP-TOPSIS for ranking additive manufacturing processes for mi-crofabrication. Rapid Prototyp. J. 2018, 24, 424-435. [CrossRef]

17. Nazam, M.; Xu, J.; Tao, Z.; Ahmad, J.; Hashim, M. A fuzzy AHP-TOPSIS framework for the risk assessment of green supply chain implementation in the textile industry. Int. J. Supply Oper. Manag. 2015, 2, 548-568.

18. Ansari, T.J.; Al-Zahrani, F.A.; Pandey, D.; Agrawal, A. A fuzzy TOPSIS based analysis toward selection of effective security requirements engineering approach for trustworthy healthcare software development. BMC Med. Inf. Decis. Mak. 2020, $20,1-13$. [CrossRef]

19. Kumar, R.; Alenezi, M.; Ansari; Gupta, B.; Agrawal, A.; Khan, R.; Prince Sultan University; Shri Ramswaroop Memorial University. Evaluating the Impact of Malware Analysis Techniques for Securing Web Applications through a Decision-Making Framework under Fuzzy Environment. Int. J. Intell. Eng. Syst. 2020, 13, 94-109. [CrossRef]

20. Alenezi, M.; Agrawal, A.; Kumar, R.; Khan, R.A. Evaluating Performance of Web Application Security Through a Fuzzy Based Hybrid Multi-Criteria Decision-Making Approach: Design Tactics Perspective. IEEE Access 2020, 8, 25543-25556. [CrossRef]

21. Coronato, A.; Paragliola, G. A structured approach for the designing of safe aal applications. Expert Syst. Appl. 2017, 85, 1-13. [CrossRef]

22. Koleva, P.; Tonchev, K.; Balabanov, G.; Manolova, A.; Poulkov, V. Challenges in designing and implementation of an effective Ambient Assisted Living system. In Proceedings of the 2015 12th International Conference on Telecommunication in Modern Satellite, Cable and Broadcasting Services (TELSIKS), Nis, Serbia, 14-17 October 2015; pp. 305-308.

23. Berger, M.; Fuchs, F.; Pirker, M. Ambient intelligence-from personal assistance to intelligent megacities. Front. Artif. Intell. Appl. 2007, 164, 21.

24. Augusto, J.C.; Shapiro, D. (Eds.) Advances in Ambient Intelligence; IOS Press: Amsterdam, The Netherlands, 2007 ; Volume 164.

25. Wartena, F.; Muskens, J.; Schmitt, L.; Petković, M. Continua: The reference architecture of a personal telehealth ecosystem. In Proceedings of the 12th IEEE International Conference on e-Health Networking, Applications and Services, Lyon, France, 1-3 July 2010; pp. 1-6. 
26. Hietala, H.; Ikonen, V.; Korhonen, I.; Lahteenmaki, K.; Maksimainen, A.; Pakarinen, V.; Saranummi, N. Feelgood-Ecosystem of Phr Based Products and Services; Research Report VTT-R-07000-09; VTT Technical Research Centre of Finland: Tampere, Finland, 2009.

27. Anouar, A.; Abdellah, T.; Abderahim, T. A novel reference model for ambient assisted living systems' architectures. Appl. Comput. Inform. 2020. [CrossRef]

28. Amina, E.M.; Anouar, A.; Touhafi, A.; Tahiri, A. Towards an SOA Architectural Model for AALPaas Design and Implimentation Challenges. Int. J. Adv. Comput. Sci. Appl. 2017, 8, 52-56.

29. Garcés, L.; Ampatzoglou, A.; Oquendo, F.; Nakagawa, E.Y. Assessment of Reference Architectures and Reference Models for Ambient Assisted Living Systems: A Systematic Literature Review; IGI Global: São Paulo, Brazil, 2017.

30. Salvi, D.; MontalvaColomer, J.B.; Arredondo, M.T.; Prazak-Aram, B.; Mayer, C. A framework for evaluating Ambient Assisted Living technologies and the experience of the universAAL project. J. Ambient Intell. Smart Environ. 2015, 7, 329-352. [CrossRef]

31. Ferro, E.; Girolami, M.; Salvi, D.; Mayer, C.; Gorman, J.; Grguric, A.; Stocklöw, C. The universaal platform for aal (ambient assisted living). J. Intell. Syst. 2015, 24, 301-319. [CrossRef]

32. Tazari, M.-R.; Furfari, F.; Ramos, J.-P.L.; Ferro, E. The PERSONA Service Platform for AAL Spaces. In Handbook of Ambient Intelligence and Smart Environments; Springer: Boston, MA, USA, 2010; pp. 1171-1199.

33. Wolf, P.; Schmidt, A.; Klein, M. SOPRANO-An extensible, open AAL platform for elderly people based on semantical contracts. In Proceedings of the 3rd Workshop on Artificial Intelligence Techniques for Ambient Intelligence (AITAmI'08), 18th European Conference on Artificial Intelligence (ECAI 08), Patras, Greece, 21-25 July 2008.

34. Wolf, P.; Schmidt, A.; Otte, J.P.; Klein, M.; Rollwage, S.; König-Ries, B.; Gabdulkhakova, A. openAAL-the open source middleware for ambient-assisted living (AAL). In Proceedings of the AALIANCE Conference, Malaga, Spain, 11-12 March 2010; pp. 1-5.

35. Adlassnig, K.P.; Blobel, B.; Mantas, J. (Eds.) Medical Informatics in a United and Healthy Europe: Proceedings of MIE 2009; IOS Press: Amsterdam, The Netherlands, 2009.

36. Haq, A.N.; Kannan, G. Fuzzy analytical hierarchy process for evaluating and selecting a vendor in a supply chain model. Int. J. Adv. Manuf. Technol. 2005, 29, 826-835. [CrossRef]

37. Huang, C.C.; Chu, P.Y.; Chiang, Y.H. A fuzzy AHP application in government-sponsored R\&D project selection. Omega 2008, 36, 1038-1052. 